\title{
Golgi SM protein Sly1 promotes productive trans-SNARE complex assembly through multiple mechanisms
}

\author{
M. Duan ${ }^{1}$, G. Gao ${ }^{3}$, D.K. Banfield ${ }^{3}$, A.J. Merz ${ }^{1,2, \S}$ \\ Departments of ${ }^{1}$ Biochemistry and ${ }^{2}$ Physiology \& Biophysics, University of \\ Washington, Seattle WA 98195-7350 \\ ${ }^{3}$ The Division of Life Science, The Hong Kong University of Science and Technology, \\ Clear Water Bay, Kowloon, Hong Kong, SAR of China
}

§Corresponding author: merza@uw.edu·+1-206-616-8308 


\section{SUMMARY}

SNARE chaperones of the Sec1/mammalian Unc-18 (SM) family have critical roles in SNAREmediated membrane fusion. Using SNARE and Sly1 mutants, and a new in vitro assay of fusion, we separate and assess proposed mechanisms through which Sly1 augments fusion: (i) opening the closed conformation of the Qa-SNARE Sed5; (ii) close-range tethering of vesicles to target organelles, mediated by the Sly1-specific regulatory loop; and (iii) preferential nucleation of productive trans-SNARE complexes. We show that all three mechanisms are important and operate in parallel, and we present evidence that close-range tethering is particularly important for trans- complex assembly when cis-SNARE assembly is a competing process. In addition, the autoinhibitory $\mathrm{N}$-terminal Habc domain of Sed5 has at least two positive activities: the Habc domain is needed for correct Sed5 localization, and it directly promotes Sly1-dependent fusion. Remarkably, "split Sed5," with the Habc domain present only as a soluble fragment, is functional both in vitro and in vivo.

\section{INTRODUCTION}

SNARE-mediated membrane fusion is central to secretory cargo transport, exocytosis, and organelle biogenesis and homeostasis (Jahn and Fasshauer, 2012; Ungar and Hughson, 2003). Fusion is preceded by tethering, mediated by a diverse group of proteins and usually controlled by small G proteins of the Rab, Arf, or Rho families (Angers and Merz, 2011; Bombardier and Munson, 2015; Pfeffer, 2017; Stenmark, 2012). Tethering is followed by docking: the assembly of a parallel, tetrahelical trans-SNARE complex ("SNAREpin") that links the two membranes (Hanson et al., 1997; Nichols et al., 1997; Sutton et al., 1998; Weber et al., 1998). "Zippering" of the incipient trans-SNARE complex does the mechanical work of driving the membranes together to initiate fusion (Zorman et al., 2014). Each trans-SNARE complex contains four $\alpha$-helices, one from each of four SNARE subfamilies: R, Qa, Qb, and Qc (Fasshauer et al., 1998). R-SNAREs often correspond to vesicle- or V-SNAREs, while Qa-SNARES (also called syntaxins) typically correspond to target membrane, or t-SNAREs. All Qa-SNAREs have in common an N-terminal regulatory "Habc" domain that folds into a trihelical bundle. In 
some but not all cases the Habc domain can fold back onto the catalytic SNARE domain to form an autoinhibited "closed" conformation (Demircioglu et al., 2014; Dulubova et al., 1999; Dulubova et al., 2001; Fernandez et al., 1998; Kosodo et al., 1998; Misura et al., 2000; Munson and Hughson, 2002; Nicholson et al., 1998; Struthers et al., 2009).

In addition to SNAREs and tethering factors, proteins of the Sec1/mammalian Unc-18 (SM) family have critical roles in SNARE-mediated fusion (Carr and Rizo, 2010; Rizo and Sudhof, 2012; Sudhof and Rothman, 2009). The first SM proteins identified through genetic screens were Vps33a (carnation in Drosophila) and Saccharomyces cerevisiae Sec1 (UNC-18 in Caenorabditis elegans; Munc18-1 or nSec1 in mammals; Novick et al., 1979; Patterson, 1932). Despite their early identification and clear importance, and despite major efforts by many laboratories, the general mechanisms of SM function are only now emerging. All SM proteins exhibit strong evolutionary and structural homology, but they interact with cognate SNARE proteins in very different ways. For example, yeast Sly1, yeast Vps45, and Munc18-1 all interact with short N-peptides at the amino termini of their cognate Qa-SNARE proteins (Bracher and Weissenhorn, 2002; Carpp et al., 2006; Dulubova et al., 2002; Furgason et al., 2009; Grabowski and Gallwitz, 1997; Yamaguchi et al., 2002). In contrast, Qa-SNARE N-peptide interactions do 45 not occur with human or yeast Vps33, or with yeast Sec1 (Baker et al., 2015; Dulubova et al., 2001; Lobingier and Merz, 2012; Togneri et al., 2006). We have called SM proteins that interact with Qa-SNARE N-peptides Class I, and those that do not, Class II (Lobingier and Merz, 2012).

Early structural and biochemical studies revealed that Munc18-1 tightly binds the QaSNARE Syntaxin-1A in its closed conformation, suggesting an inhibitory role for Munc18-1. (Dulubova et al., 1999; Misura et al., 2000; Yang et al., 2000). However, the emerging consensus is that the core and evolutionarily conserved role of SM proteins is positive, rather than inhibitory. Specifically, SM proteins are hypothesized to nucleate and stabilize fusioncompetent trans-SNARE complexes (Carr and Rizo, 2010; Sudhof and Rothman, 2009; Toonen and Verhage, 2003; Yoon and Munson, 2018). A breakthrough was achieved in 2015 with two structures of yeast Vps33: one with a Qa-SNARE domain bound, and another with an R-SNARE domain bound (Baker et al., 2015, \#55487). When superimposed these structures implied that Vps33 templates the initial assembly of the trans-SNARE complex, allowing the trans-complex to transit into a metastable "half-zipped" intermediate. Single-molecule force spectroscopy 
experiments strongly supported this interpretation (Jiao et al., 2018; Ma et al., 2015). It remains unclear whether the templating mechanism is generalizable to all SMs, and it is unclear to what extent SMs promote SNARE-mediated fusion through additional general or pathway-specific mechanisms.

We have turned our attention to Sly1, the ER-Golgi SM. Sly1 has been proposed to promote fusion through several different mechanisms. First, meticulous solution biochemistry demonstrated that Sly1 can open the inactive closed conformation of the cognate Qa-SNARE Sed5. This in turn allows Sed5 to more readily complex with Qb, Qc, and R-SNAREs (Bos1, Bet1, and Sec22; (Demircioglu et al., 2014; Kosodo et al., 1998). A limitation of this work is that the SNAREs used were soluble fragments; the roles of Sed5 inhibition and opening were not tested in experiments that assayed membrane fusion. Second, we demonstrated that Sly1 binding to quaternary SNARE complexes in solution slows the kinetics of ATP-dependent disassembly by Sec17 and Sec18 (in mammals, $\alpha$-SNAP and NSF). Consistent with these findings, in vivo genetic tests revealed that Sec17 overproduction is tolerated in a wild-type genetic background but becomes lethal when Sly1 function is partially compromised (Lobingier et al., 2014). Third, on the basis of structural homology to Vps33, Baker et al. (2015) proposed that Sly1 can template Qa- and R-SNARE trans-complex formation. Fourth, experiments in a companion manuscript (Duan et al., submitted) demonstrate that Sly1 can promote close-range vesicle tethering through an amphipathic helix, $\alpha 21$, that directly interacts with the vesicle membrane.

All of these mechanisms are plausible, yet no study to date has attempted to assess their functional contributions within a unified experimental framework. Here we begin that effort, combining in vivo genetic tests with a new chemically defined in vitro reconstitution of fusion on the ER-Golgi anterograde pathway. Focusing on Sly1 interactions with the Qa-SNARE Sed5, we demonstrate that multiple mechanisms do indeed contribute to Sly1's fusionpromoting activity and, unexpectedly, that the regulatory Habc domain of the Qa-SNARE Sed5 augments Sly1-stimulated fusion, rather than being solely auto-inhibitory. 


\section{RESULTS}

\section{Sed5 $\mathbf{N}$-peptide is essential for viability and efficient membrane fusion}

The Qa-SNARE Sed5 has four domains: an N-peptide of 21 residues that binds tightly to Sly1; a trihelical Habc domain that is auto-inhibitory; the Qa-SNARE domain; and a C-terminal transmembrane segment (Fig. 1A). In a previous study, missense mutations that reduced the affinity of Sly1 for the N-peptides of its client Qa-SNAREs (Sed5 and Ufe1) resulted in minimal defects in assays for viability, secretion, and Sly1 localization. These results were interpreted to indicate the functional "irrelevance" of Sly1-Sed5 interactions (Peng and Gallwitz, 2004). In contrast to these findings, overexpression of a Sly1 cognate N-peptide, or of the Sly $1 \mathrm{~N}$ peptide binding domain, shattered the Golgi in mammalian cells (Dulubova et al., 2003; Yamaguchi et al., 2002). To reassess whether the Sed5 N-peptide is functionally important in budding yeast we engineered an allele, sed5 $\Delta N$, that encodes a Sed5 variant lacking the Npeptide (as defined by the crystal structure PDB 1MQS; Bracher et al., 2002; Fig. 1A). In a genetic background expressing wild type $S L Y 1$, sed $5 \Delta N$ was a recessive lethal allele (Fig. 1B;

Supplementary Fig. S1). Thus, the Sed5 N-peptide is essential for viability. These results are buttressed by recent experiments showing that sed5(DFTV), a quadruple missense mutant

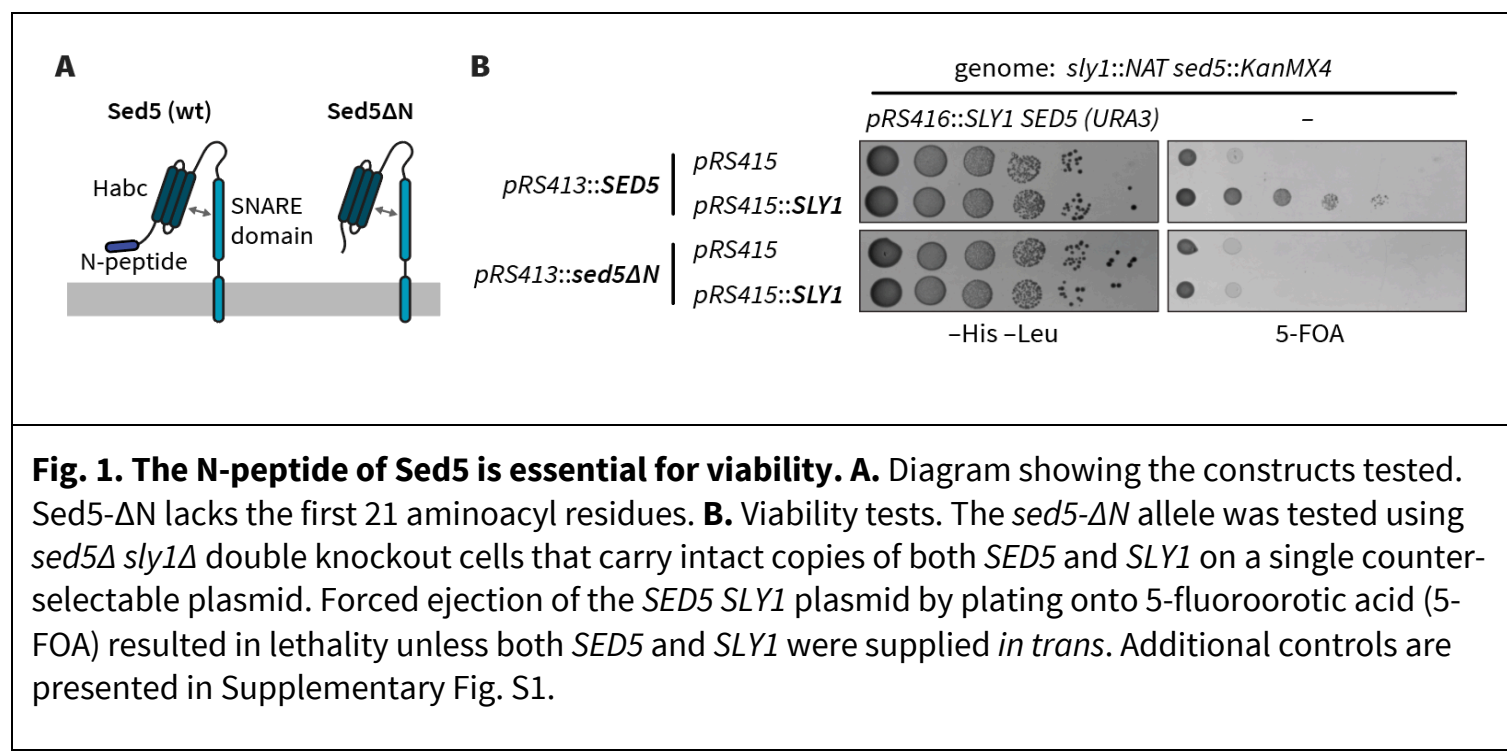


that impairs Sly1 binding to the Sed5 N-peptide, is also recessive lethal (Gao and Banfield, 2019).

To test whether the Sed5 N-peptide has a direct role in Sly1-dependent fusion we used a chemically defined assay of fusion driven by ER-Golgi SNAREs (Duan et al., submitted; Zucchi and Zick, 2011). Briefly, we prepare reconstituted proteoliposomes (RPLs) bearing ER-Golgi SNARES, with two orthogonal FRET reporter pairs. These reporters simultaneously monitor lipid and content mixing in a single $20 \mu \mathrm{L}$ reaction. Here, we present content mixing results (the reaction endpoint). Fusion requires the presence of Sly1 and also depends on 3\% polyethylene glycol, a molecular crowding agent that mimics the action of tethering factors (Duan et al., submitted; Furukawa and Mima, 2014; Lentz, 2007; Mitchison, 2019; Yu et al., 2015). Sly1-mediated fusion is further stimulated by the universal SNARE disassembly chaperones Sec17 ( $\alpha$-SNAP), Sec18 (NSF), and $\mathrm{Mg}^{2+} \cdot$ ATP (Duan et al., submitted).

To test the function of the N-peptide we prepared RPLs bearing either wild-type Sed5 or Sed5 $\Delta \mathrm{N}$ (lacking the first 21 aminoacyl residues), as well as the Qb- and Qc-SNAREs Bos1 and Bet1. These Q-SNARE RPLs were assayed for their ability to fuse with RPLs bearing the RSNARE Sec22. In reactions containing Sec17, Sec18, and $\mathrm{Mg}^{2+} \cdot$ ATP, fusion was rapid and efficient when both 3\% PEG and Sly1 were present. However, both the rate and extent of fusion were dramatically reduced when RPLs bearing $\operatorname{Sed} 5 \Delta \mathrm{N}$ were tested. Moreover, high concentrations of Sly1 were required to stimulate fusion of Sed5 $\Delta N$ RPLs. With wild type Sed5 near-maximal fusion was observed at $100 \mathrm{nM}$ Sly1, while with Sed5 $\Delta \mathrm{N}$ the rate and extent of fusion were much lower even at 1600 nM Sly1 (compare Figs. 2C and 2D). When PEG(which promotes vesicle tethering) was omitted (Fig. 2F), fusion with $\operatorname{Sed} 5 \Delta \mathrm{N}$ was undetectable even when 1600 nM Sly1 was added. We conclude that the Sed5 N-peptide strongly promotes Sly1dependent fusion, both in vivo and in vitro. 


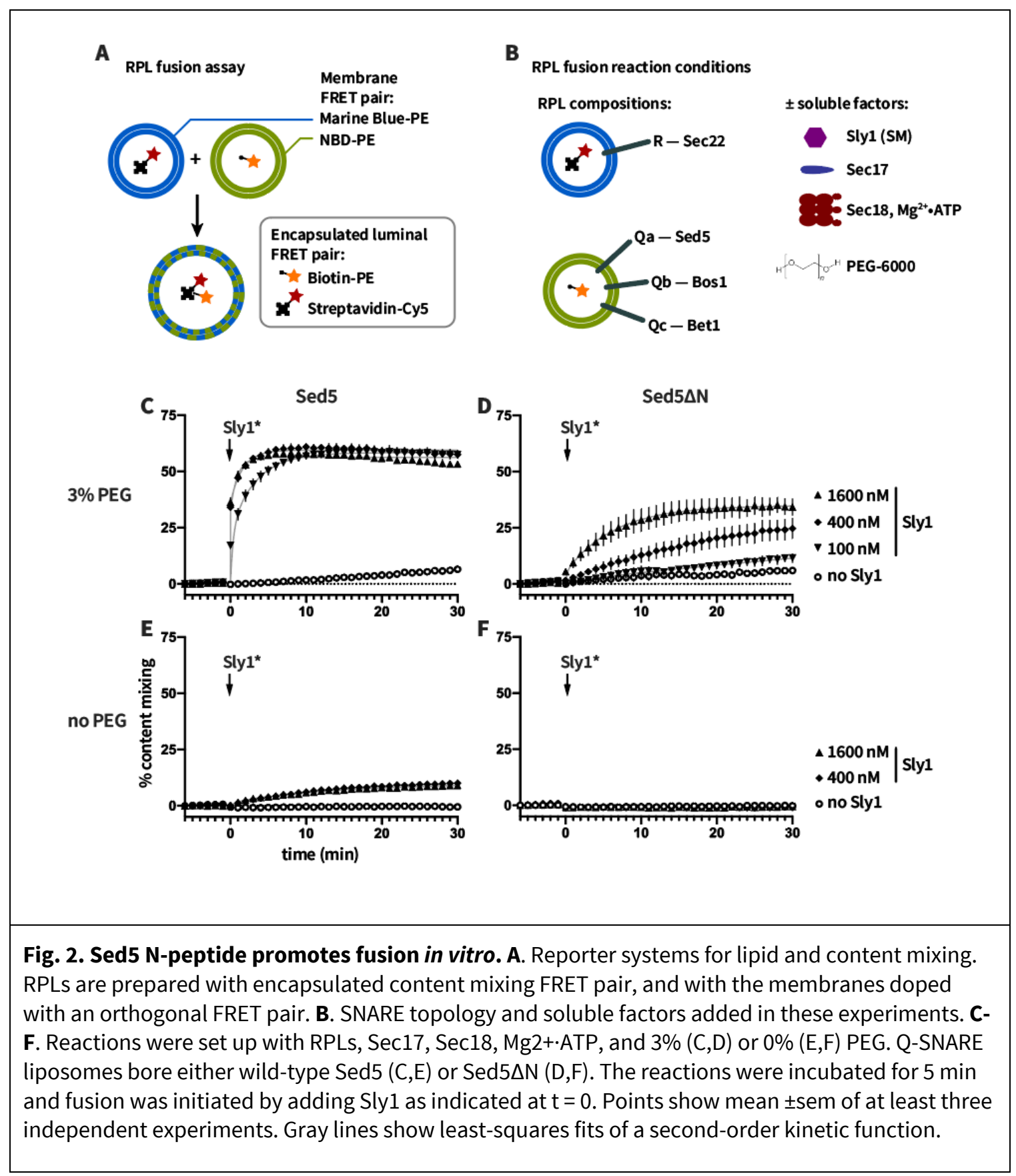

\section{Sly1 hyperactivity suppresses lethality of sed5 $\Delta N$ and restores fusion}

The hyperactive allele $S L Y 1-20$ was initially identified as a dominant, single-copy suppressor of

130 loss of Ypt1, the yeast Rab1 homolog (Dascher et al., 1991; Ossig et al., 1991). Single-copy

$S L Y 1-20$, or multi-copy wild type $S L Y 1$, were subsequently found to suppress deficiencies of a 
wide variety of proteins that mediate intra-Golgi, ER-Golgi, and Golgi-ER vesicle docking and fusion. In the companion paper (Duan et al., submitted) we show that the mechanism of Sly120 hyperactivity involves both release of Sly1 autoinhibition and coupled activation of a latent vesicle tethering activity within the Sly1 auto-inhibitory loop. Somewhat surprisingly, SLY1-20 was able to suppress the lethal phenotype of $\operatorname{sed} 5 \Delta N$, but only when $S L Y 1-20$ was provided on a multiple-copy plasmid (Fig. 3A). Single-copy $S L Y 1-20$, or multiple copy wild-type $S L Y 1$, were unable to rescue the growth of sed5 $\Delta N$ mutant cells. Thus, the sed5 $\Delta N$ allele is even more deleterious than the already-lethal ypt1-3 (Rab1-deficient) or uso1 (p115/Uso1 tethering factor-deficient) alleles - both of which are efficiently suppressed by single-copy SLY1-20. The survival of $\operatorname{sed} 5 \Delta N$ cells containing high-copy $S L Y 1-20$ also allowed us to verify that $\operatorname{Sed} 5 \Delta N$ is present at normal abundance and migrates as expected on SDS-PAGE gels (Fig. 3B).

Genetic suppression can occur through direct or indirect mechanisms. Thus, we used the RPL system to test whether suppression of sed5 $\triangle N$ by multicopy SLY1-20 occurs through a direct or indirect mechanism. In the presence of PEG, Sly1-20 at $1600 \mathrm{nM}$ was able to drive fusion of Sed5 $\triangle N$ RPLs to nearly the rates and extents seen with wild type Sed5 RPLs and wild type Sly1 at 100 nM (compare Figs. 3C and D). However, in the absence of PEG (that is, under tethering-deficient conditions), Sly1-20 was unable to drive fusion of RPLs bearing Sed5 $\Delta N$, even at the highest concentrations tested (Fig. 3E,F). Taken together, the fusion results closely mirror the in vivo matrix of genetic interactions among SED5 and SLY1 alleles. When wild-type Sly1 is present, fusion is severely attenuated if the Sed5 $\mathrm{N}$-peptide is deleted, but high concentrations of Sly1-20 can rescue Sed5 $\Delta 21$. The in vitro experiments show that this rescue occurs through direct effects on the fusion machinery. With wild-type Sed5, Sly1-20 at moderate concentrations can compensate for tethering deficiencies either in vitro (0\% PEG) or in vivo (e.g., ypt1 or uso1 deficiency). However, consistent with in vitro tethering assays (Duan et al., submitted), our fusion experiments show that hyperactive Sly1-20 cannot compensate simultaneously for loss of the Sed5 N-peptide and reduced tethering. 


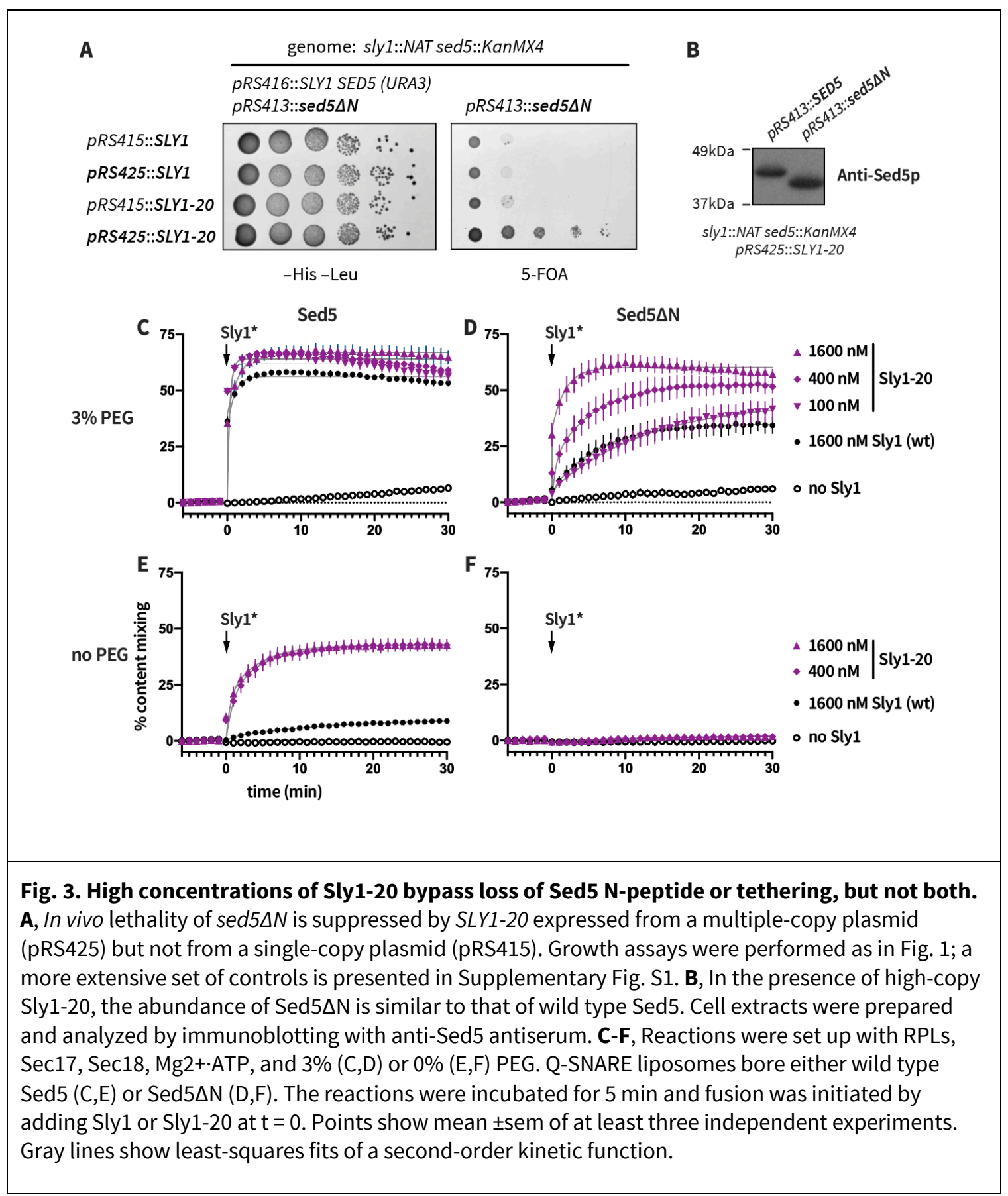




\section{Sly1 can stimulate fusion independently of Sed5 opening}

Sly1 is reported to open closed Sed5, to allow assembly of SNARE core complexes (Demircioglu et al., 2014). We hypothesized that Sed5 opening might be only one of multiple mechanisms through which Sly1 stimulates fusion. To test this idea, we prepared RPLs bearing two different Sed5 mutants that cannot adopt a closed conformation (Fig. 4A). Sed5 $\Delta \mathrm{Habc}$ lacks the autoinhibitory Habc domain required to form a closed conformation, but still has the N-terminal 21 amino acids which bind Sly1 with high affinity. Sed5 $\Delta \mathrm{N}-\mathrm{Habc}$ lacks both the N-peptide and the Habc domain.

In vivo, both sed5 $\Delta H a b c$ and sed5 $\Delta \mathrm{N}-\mathrm{Habc}$ confer recessive lethal phenotypes. The lethality of these alleles was not suppressed by expression of $S L Y 1$ or $S L Y 1-20$, from either single- or multiple-copy vectors (Supplementary Fig. S2A). Analyses in SED5/sed5 cells indicated that the protein products of the sed5 mutant alleles are indeed synthesized (Supplementary Fig. S2B). However, these mutant proteins are mis-localized and ultimately degraded in the lumen of the lysosomal vacuole (Supplementary Fig. S2C). The Habc domain therefore contains information essential for correct Sed5 localization and in vivo function. Two of us have recently performed a more detailed analysis of Sed5 localization determinants (Gao and Banfield, 2019).

To assess the role of Sed5 autoinhibition in membrane fusion we returned to the in vitro RPL assay system. As before, RPLs bearing wild type Sed5 or Sed5 $\Delta \mathrm{N}$ exhibited little or fusion when Sly1 was absent (Fig. 4 B,C; black open circles). In contrast, RPLs bearing either Sed5 $\Delta \mathrm{Habc}$ or Sed5 $\Delta \mathrm{N}-\mathrm{Habc}$ exhibited spontaneous but slow Sly1-independent fusion (Fig. 4D,E; black open circles). These results strongly corroborate solution biochemistry studies which indicate that autoinhibition by the Sed5 Habc domain prevents SNARE core complex assembly (Demircioglu et al., 2014). In the presence of Sly1 or Sly1-20, however, fusion was stimulated whether the Habc domain was present (Fig. 4B,C) or absent (Fig. 4D,E). We conclude that although one function of Sly1 is to open the closed conformation of Sed5, Sly1 must also have additional fusion-promoting activities. 


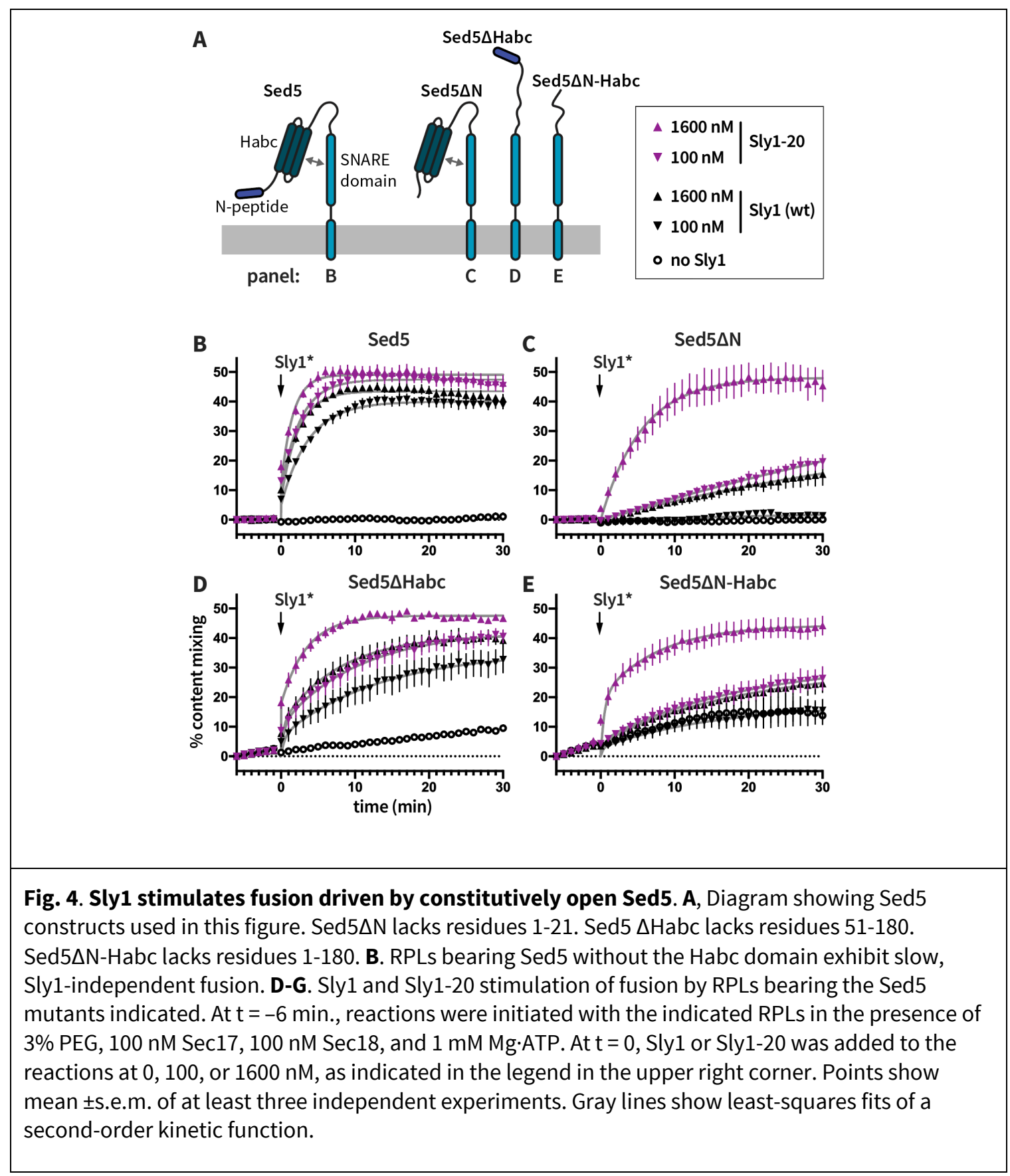




\section{tethering}

An auto-inhibitory loop conserved among Sly1 family members harbors a close-range vesicle tethering activity, and this activity is indispensable for the hyperactivity of the Sly1-20 mutant (Duan et al., submitted). We therefore asked whether Sly1 can stimulate fusion independently of both its close-range tethering function and its ability to open the Sed5 closed conformation. Reactions were initiated with RPLs bearing Sed5 $\Delta \mathrm{Habc}$ (which cannot adopt a closed conformation), and with wild type Sly1 or Sly1 mutants defective in close-range tethering (Fig. 5A). The Sly1 1 loop mutant lacks the entire Sly1-specific regulatory loop including the amphipathic helix $\alpha 21$, which is required for close-range membrane tethering. When Sly $1 \Delta$ loop was added to reactions containing Sed5- $\Delta$ Habc RPLs, an increase in fusion was still observed, indicating that Sly1 must have fusion-stimulating activities beyond Sed5 opening and close-range tethering.

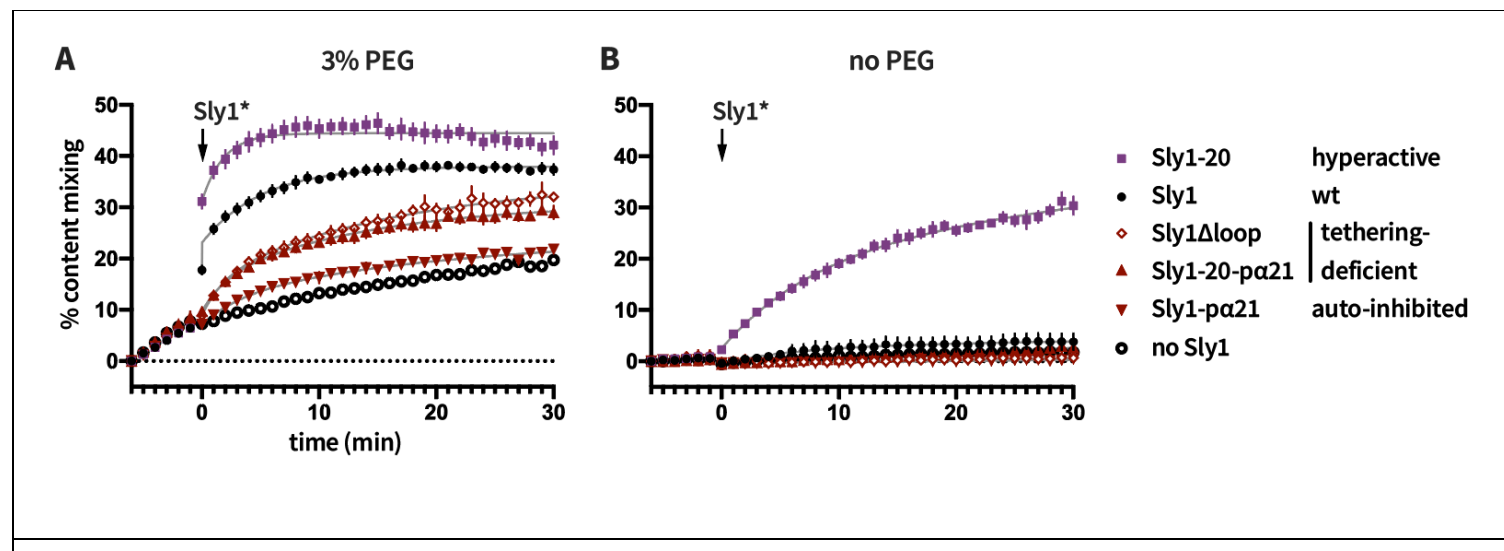

Fig. 5. Sly1 can stimulate fusion independently of both Sed 5 opening and close-range tethering. At $\mathrm{t}=-6$ min., reactions were initiated with R-SNARE RPLs, Q-SNARE RPLS bearing Sed5 $\Delta \mathrm{Habc}, 100 \mathrm{nM}$ Sec17, $100 \mathrm{nM}$ Sec18, and $1 \mathrm{mM}$ Mg.ATP. The reactions also contained (A) 3\% PEG or (B) $0 \%$ PEG. At t =0, the indicated Sly1 variants were added to $1600 \mathrm{nM}$ final, as indicated in the legend. Points show mean \pm sem of at least three independent experiments. Gray lines show least-squares fits of a second-order kinetic function.

This conclusion was buttressed by two additional Sly1 mutants. In the Sly1pa21 protein, five apolar residues within helix $\alpha 21$ are mutated, preventing the loop from binding to membranes. Sly1-pa21 has at least two functional defects: it is constitutively auto-inhibited, and it is defective for close-range tethering (Duan et al., submitted). As expected, Sly1-pa21 stimulated only barely detectable fusion above background (Fig. 5A). In the compound 
mutant Sly1-20-pa21, autoinhibition is released (the loop is open), but close-range tethering is still compromised. When added to reactions with Sed5 $\Delta$ Habc RPLs, Sly1-20-pa21 stimulated fusion similarly to Sly1 $\Delta$ loop (Fig. 5A). When the same panel of Sly1 variants was tested with Sed5 $\triangle$ Habc RPLs under tethering-deficient conditions (Fig. 5B), only Sly1-20 (constitutively open and presenting helix $\alpha 21$ ) was able to stimulate substantial fusion. Thus, fusion of Sed5 $\triangle \mathrm{Habc}$ RPLs requires a tethering activity which can be provided either by the tetheringhyperactive Sly1-20, or by PEG. We conclude that both Sly1 opening of closed Sed5, and Sly1 close-range tethering activity, contribute to the ability of Sly1 to promote fusion, and that Sly1 has one or more additional fusion-promoting activities. In Vps33 and Munc18-1, domain 3a appears to serve as a template for trans-SNARE complex assembly (Baker et al., 2015; Jiao et al., 2018). The near-inability of the constitutively auto-inhibited mutant Sly1-pa21 to stimulate fusion (Fig. 5A) implies that the additional Sly1 activities involve Sly1 domain 3a, which is occluded when Sly1 is auto-inhibited (Baker et al., 2015; Bracher and Weissenhorn, 2002).

\section{Close-range tethering by Sly1 promotes assembly of trans-SNARE complexes}

Assembly of cis-SNARE complexes occurs spontaneously and competes with assembly of fusion-active trans-complexes. To test the hypothesis that close-range tethering by Sly 1 specifically favors trans-SNARE complex assembly, we evaluated the inhibitory activity of the soluble R-SNARE domain of Sec22 (Sec22 $2_{S N}$-GFP; Fig. 6). Reactions were initiated with RPLS bearing the three Q-SNARES along with $\operatorname{Sec} 22_{S N}-G F P(0,2$ or $20 \mu \mathrm{M})$, and wild type or mutant forms of Sly1. The reactions were incubated for $15 \mathrm{~min}$, then R-SNARE RPLs were added and the reactions were incubated for an additional $6 \mathrm{~min}$. To initiate fusion PEG was added $(t=0)$. PEG was added to $4 \%$ final rather than $3 \%$ (as in previous experiments) in an effort to compensate for the tethering defects of the Sly1-20- $\alpha 21 \mathrm{p}$ and Sly1 $\Delta$ loop mutants. Reactions driven by wild-type Sly 1 or hyperactive Sly1-20 were partially inhibited by $2 \mu \mathrm{M}$ Sec $22_{\mathrm{SN}_{\mathrm{N}}}$-GFP (Fig. 6B), and almost totally inhibited by $20 \mu \mathrm{M} \mathrm{Sec} 22_{\mathrm{SN}}$-GFP (Fig. 6C). In contrast, reactions containing Sly1-20- $\alpha 21 \mathrm{p}$ or Sly1 $\Delta$ loop were almost totally inhibited at both 2 and $20 \mu \mathrm{M}$ Sec22 $2_{S N}-$ GFP (Fig. 6B,C). This indicates that reactions driven by tethering-deficient Sly1 mutants are more sensitive to inhibition by cis-complex assembly. 


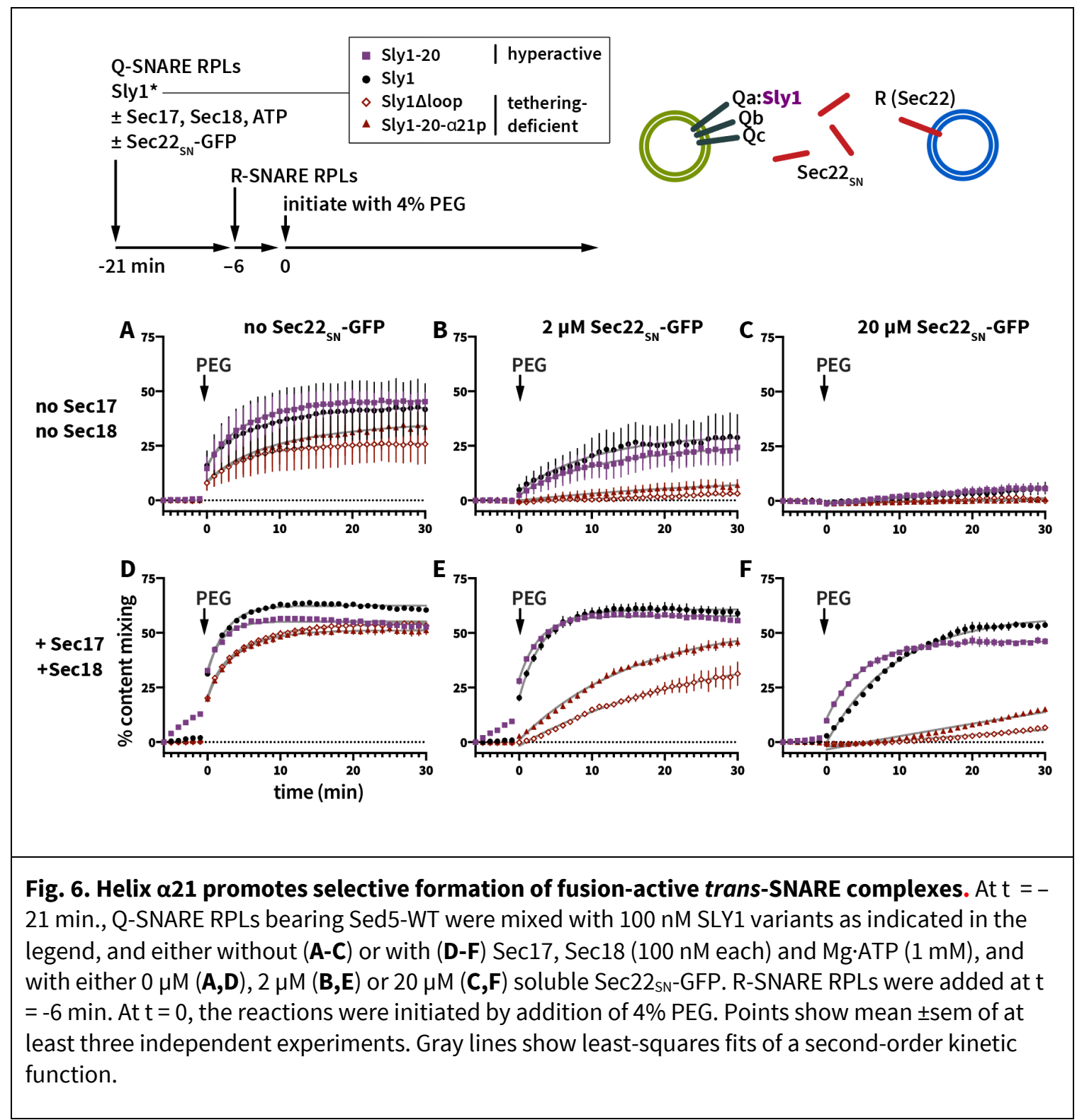

We next tested whether inhibition of fusion by Sec22 $2_{S N}$-GFP can be reversed by driving cycles of SNARE complex disassembly, to generate free SNAREs on the RPLs. Reactions were initiated as in Fig. 6A-C, but with $\mathrm{Sec} 17, \operatorname{Sec} 18$, and $\mathrm{Mg}^{2+}$.ATP (Fig. 6D-F). Reactions containing Sly1 or hyperactive Sly1-20 exhibited efficient fusion in the presence of Sec17/18, even at $20 \mu \mathrm{M}$ Sec $22_{S N}-$ GFP. In contrast, reactions driven by the tethering deficient Sly1 mutants exhibited far less rescue. Similar results were obtained when reactions were run under the same conditions, but with 3\% rather than 4\% PEG (Supplementary Fig. S3). Taken together these experiments support the hypothesis that $\alpha 21$-mediated tethering favors assembly of fusogenic trans-SNARE complexes versus non-fusogenic cis-SNARE complexes, even when the 
pool of unpaired SNAREs is continuously replenished through cycles of Sec17- and Sec18mediated cis-SNARE complex disassembly.

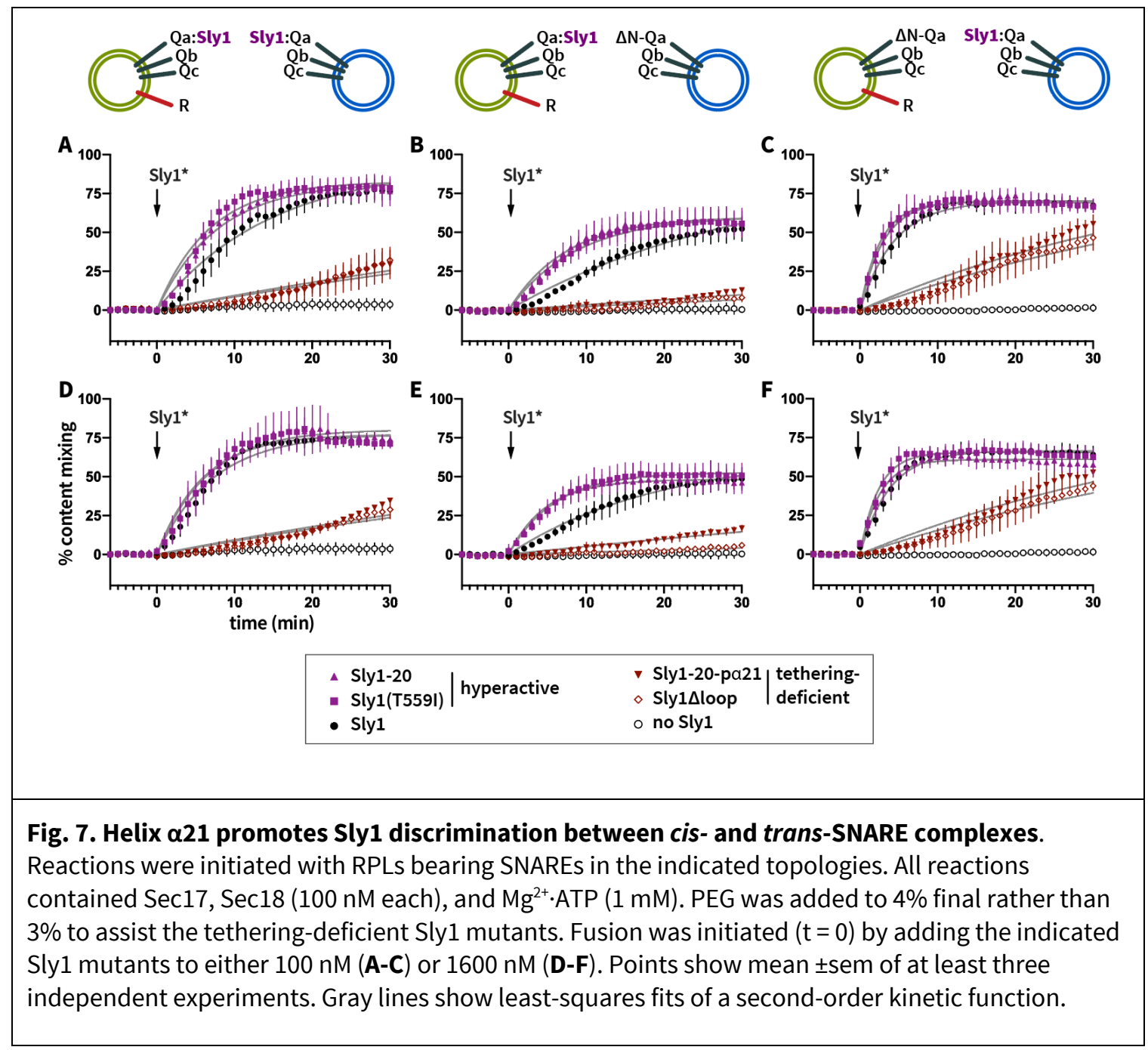

We wondered whether similar results might be obtained under more physiologically relevant conditions, using wild-type membrane-anchored Sec22 rather than soluble Sec22. We therefore assayed the fusion of 4-SNARE QabcR RPLs with 3-SNARE Qabc RPLs, in the presence of wild type and mutant Sly1 variants (Fig. 7). Because QabcR RPLs can form quaternary SNARE complexes in cis as well as in trans, they require Sec17/18 for efficient fusion. In this configuration the formation of trans-SNARE complexes and the re-formation of cis-SNARE complexes are competing processes. As above, we ran reactions in the presence of $4 \% \mathrm{PEG}$ in an effort to compensate for the defects of tethering-deficient Sly1 mutants. Even given the results with soluble Sec22, we were surprised at the clarity of the results: Sly1-20- $221 \mathrm{p}$ and 
Sly1 1 loop were almost fusion-inactive when confronted with the QabcR SNARE topology (Fig. 7A), even when the Sly1 mutants were supplied at $1600 \mathrm{nM}$ (Fig. 7D) and despite the presence of $4 \%$ PEG. These defects are considerably more severe than the defects observed in RPL experiments using the Qabc vs. R-SNARE topology, where QabcR cis-SNARE complexes cannot form during the first round of fusion (e.g., Fig. 6a; and Duan et al., submitted, Fig. 5B,D). Two mutants hyperactive for close-range tethering , Sly1-20 and Sly1-T559I (Duan et al., submitted), exhibited activity as strong as or stronger than the wild type.

As Sly1 is recruited to the Qa-SNARE Sed5 through a high-affinity interaction with the Sed5 $\mathrm{N}$-peptide, we used the Sed5 $\Delta \mathrm{N}$ mutant protein to direct Sly1 primarily to either the QabcR RPLs (Fig. 7B,E), or to the Qabc RPLs (Fig. 7C,F). The results show that fusion occurs most rapidly when Sly1 is placed in trans to the R-SNARE, Sec22 (Fig. 7C,F) - the configuration where R-SNARE binding in trans is most likely and where cis QabcR complex assembly is least likely to be stimulated by Sly1. Fusion is slowest when Sly1 is placed in cis to the R-SNARE; under this condition, the tethering-deficient Sly1 mutants are almost totally unable to drive fusion (Fig. 7C,D). Taken together the experiments in Figs. 6 and 7 demonstrate that the Sly1 close-range tethering activity takes on special importance when Sly1 must selectively catalyze formation of trans-SNARE complexes in competition with formation of inactive cis-SNARE complexes.

\section{Soluble Sed5 Habc domain promotes Sly1-stimulated fusion in vitro}

In our experiments comparing various Sed5 mutants, we were surprised to see that although Sed5 $\Delta \mathrm{Habc}$ can catalyze spontaneous Sly1-independent fusion, its ability to support Sly1stimulated fusion was reduced compared to wild type Sed5 (compare Figs. 4B and 4D). These results suggested that the Sed5 Habc domain, in addition to being autoinhibitory, might have a positive, fusion-promoting activity. To test this hypothesis we asked whether the Sed5 Habc domain, supplied in soluble form, would alter the ability of Sly1 and its cognate SNAREs to drive fusion.

Reactions were initiated with Qabc-SNARE RPLs bearing four different Sed5 variants: wild-type, Sed $5 \Delta \mathrm{N}$, Sed5 $\Delta \mathrm{Habc}$ or Sed5 $\Delta \mathrm{N}-\mathrm{Habc}$. Each of these reactions was performed in the absence or presence of soluble Sed5 Habc or N-Habc (Fig. 8A-D). Because Sly1 binds to soluble N-Habc domain with sub-nanomolar affinity (Demircioglu et al., 2014), Sly1 and 
soluble Habc or N-Habc were pre-mixed before they being added together to the reaction mixture. The reactions were initiated without PEG and monitored for $6 \mathrm{~min}$. To initiate fusion, PEG was added to $3 \%(t=0)$. To our surprise, both the Habc and $\mathrm{N}$-Habc domains of Sed5 stimulated fusion in a dose-dependent manner. Fusion was most efficiently stimulated when the N-peptide was present on the soluble Habc domain (Fig. 8B), rather than on the membrane-bound Sed5 (Fig. 8C), and less efficiently stimulated when N-peptides were present on both Sed5 and the soluble N-Habc protein (Fig. 8A). However, Habc stimulated fusion under every condition tested, even when the N-peptide was absent from both Sed5 and the soluble Habc domain (Fig. 8D).

Similar results were obtained in reactions lacking (Fig. 8A-D) or containing Sec17 and Sec18 (Fig. 8E-H). Remarkably, in the presence of Sec17 and Sec18 and at the highest concentration of $\mathrm{N}-\mathrm{Habc}$, robust fusion was observed even before PEG was added to the reaction (Fig. 8E,F; red asterisks). This indicates bypass of the tethering requirement $-\mathrm{a}$ phenotype previously observed only with hyperactive Sly1 mutants such as Sly1-20 or at very high concentrations of PEG (Duan et al., submitted). We conclude that the Sed5 Habc domain augments the efficiency of Sly1-stimulated fusion, that the Habc domain need not be covalently coupled to Sed5. In other words, "split Sed5" can drive fusion. 
bioRxiv preprint doi: https://doi.org/10.1101/2020.01.16.909630; this version posted January 16, 2020. The copyright holder for this preprint (which was not certified by peer review) is the author/funder, who has granted bioRxiv a license to display the preprint in perpetuity. It is made available under aCC-BY-NC-ND 4.0 International license.

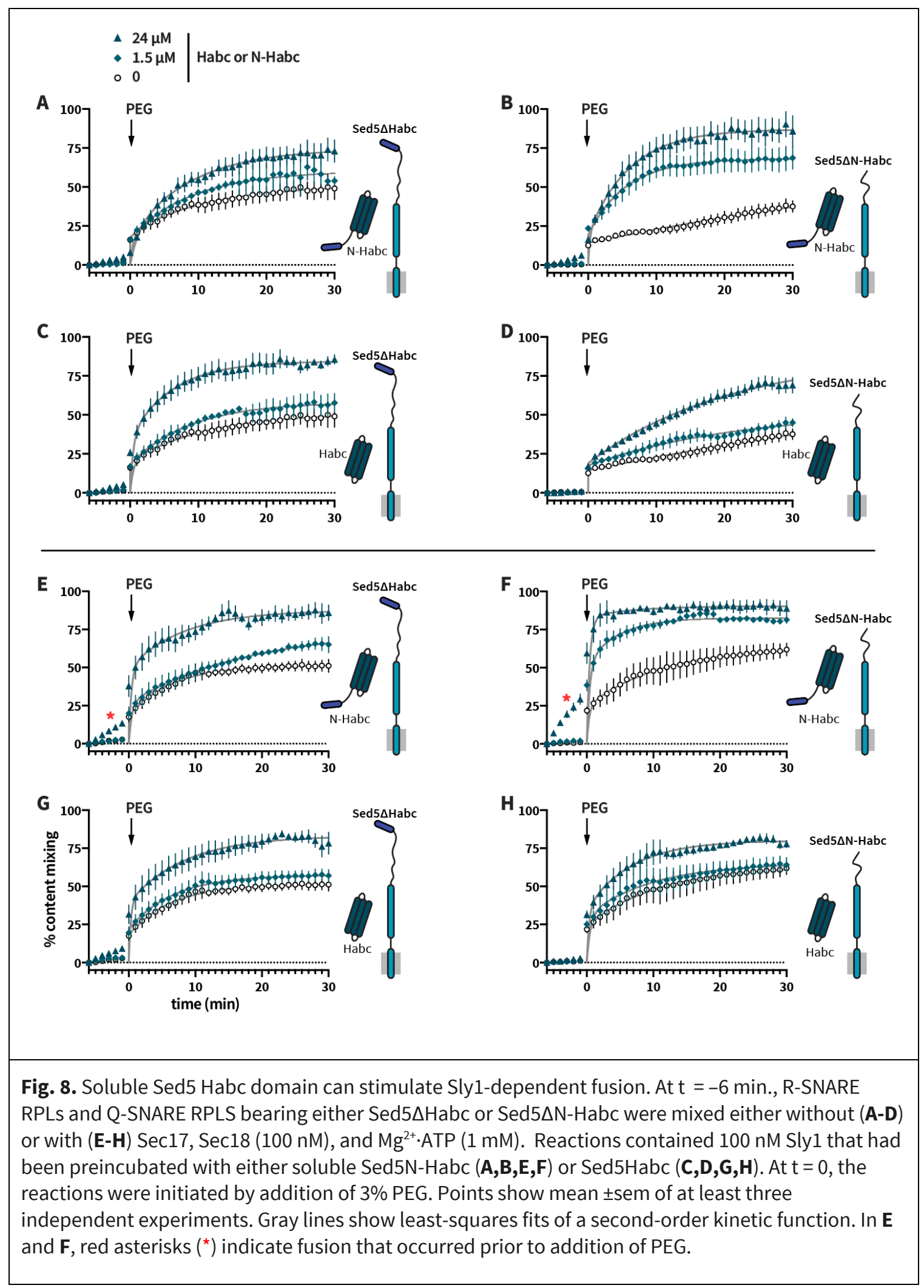




\section{Split Sed5 can function in vivo}

Next, we tested whether soluble Sed5 Habc or N-Habc domains might suppress the lethal phenotype of cells expressing Sed5 variants lacking the Habc domain (Fig. 9). Single-copy dicistronic plamids were constructed bearing sed5 $\Delta \mathrm{N}-\mathrm{Habc}$ or sed5 $\mathrm{H} \mathrm{Habc}$, as well as either $\mathrm{Habc}$ or $N$-Habc. These test plasmids were introduced into sly $1 \Delta$ sed $5 \Delta$ strains harboring a counterselectable SLY1 SED5 balancer plasmid. In vitro, we had noted that Qabc RPLs bearing wildtype Sed5 fuse with similar efficiency when either Sly1 or hyperactive Sly1-20 are supplied, but that RPLs bearing Sed5 $\Delta \mathrm{Habc}$ are considerably more responsive to Sly1-20 (Fig. 4). Thus, we also tested the effects of single-copy or multiple-copy plasmids bearing either SLY1 or SLY1-20. The results show that the Sed5 N-Habc domain can support viability when present solely as a soluble fragment (Figs 9A,B). However, viability of these "split Sed5" cells requires Sly1 hyperactivity. Only SLY1-20 expressed from a high-copy vector supported robust growth with split Sed5. Single-copy SLY1-20 facilitated very slow growth. Moreover, as in the in vitro assays, rescue was most robust when the N-peptide is on the soluble Habc fragment (Figs9 A,B). No rescue was observed in cells totally lacking the Sed5 N-peptide (Fig. 9C) and rescue was only barely detectable when the $\mathrm{N}$-peptide was present solely on the membrane-anchored mutant Sed5 (Fig. 9D).

Immunoblot analyses of whole cell lysates from cells expressing wild-type Sed5 indicated that the the truncated Sed5 variants and soluble fragments were expressed (Supplementary Fig. S4A). In cells lacking the SLY1 SED5 balancer plasmid and expressing either Sed5 $\Delta \mathrm{N}-\mathrm{Habc}$ or Sed5 $\Delta \mathrm{Habc}$, the steady-state level of the soluble N-Habc fragment depended on the gene dosage of $S L Y 1-20$, suggesting that the stability of the soluble fragment is controlled by its interaction with Sly1-20 protein (Supplementary Fig. S4B). Taken together, the in vitro and in vivo results here and in (Gao and Banfield, 2019) indicate that, in addition to being auto-inhibitory, the Sed5 Habc domain has positive functions: it both promotes Sly1-dependent membrane fusion, and regulates Sed5 subcellular localization. 


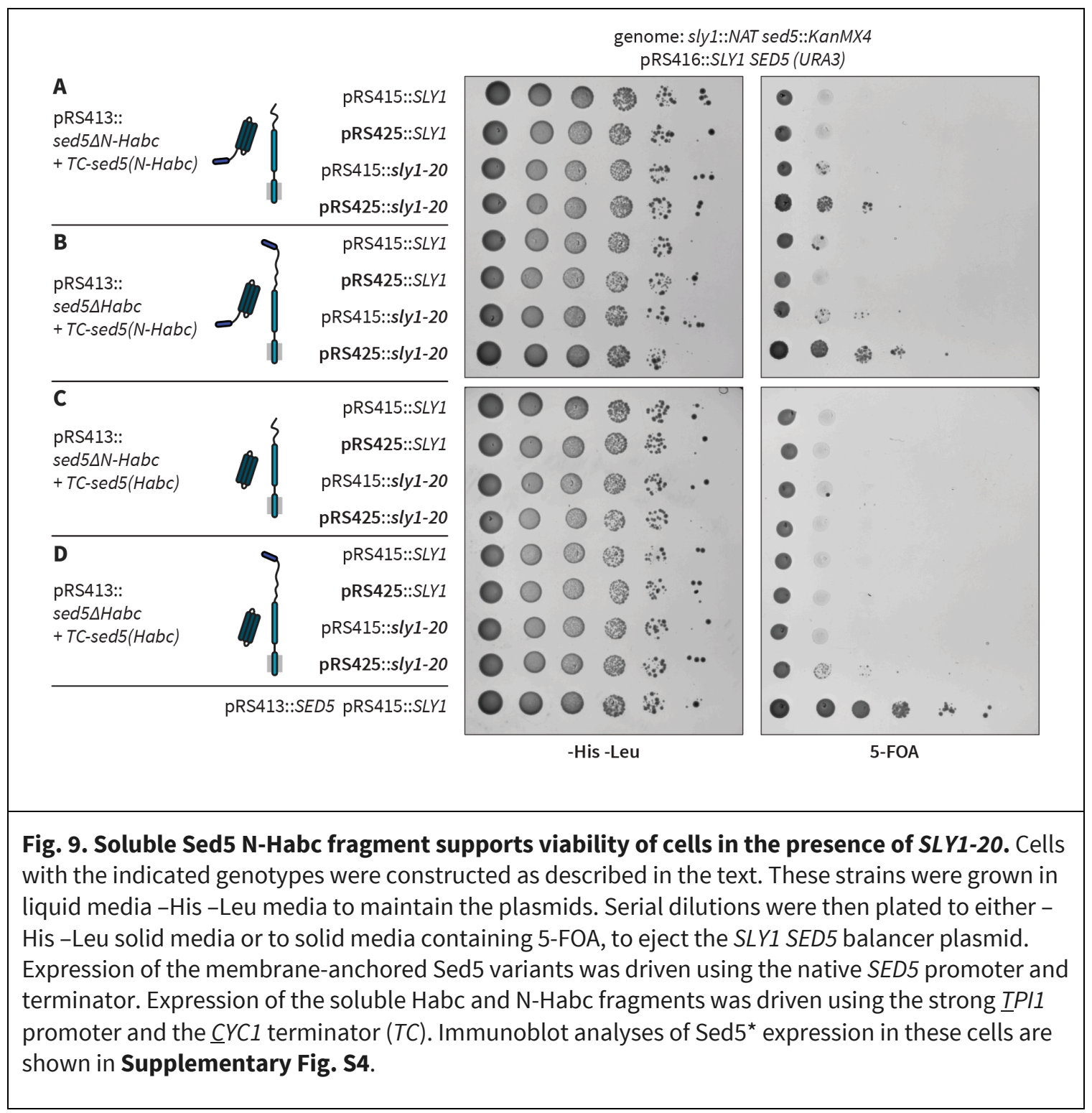




\section{DISCUSSION}

Our experiments show that Sly1 has multiple distinguishable activities which promote SNAREmediated fusion (Fig. 9). First, Sed5-bound Sly1 has the intrinsic ability to tether incoming vesicles through the amphipathic helix $\alpha 21$ within the Sly1 regulatory loop (Duan et al., submitted). Second, Sly1 has the ability to open the closed, auto-inhibited conformation of the Qa-SNARE Sed5 (Demircioglu et al., 2014). When these activities are experimentally bypassed, Sly1 still promotes fusion (albeit less efficiently) through a third activity that probably involves domain 3a. We infer that the third function is probably the selective and accurate nucleation of trans-SNARE complex assembly. These functions are interlinked. Defects in the Sly1 close-range tethering function result in dramatically impaired fusion when cis-SNARE and trans-SNARE complex assembly are competing processes, indicating that the

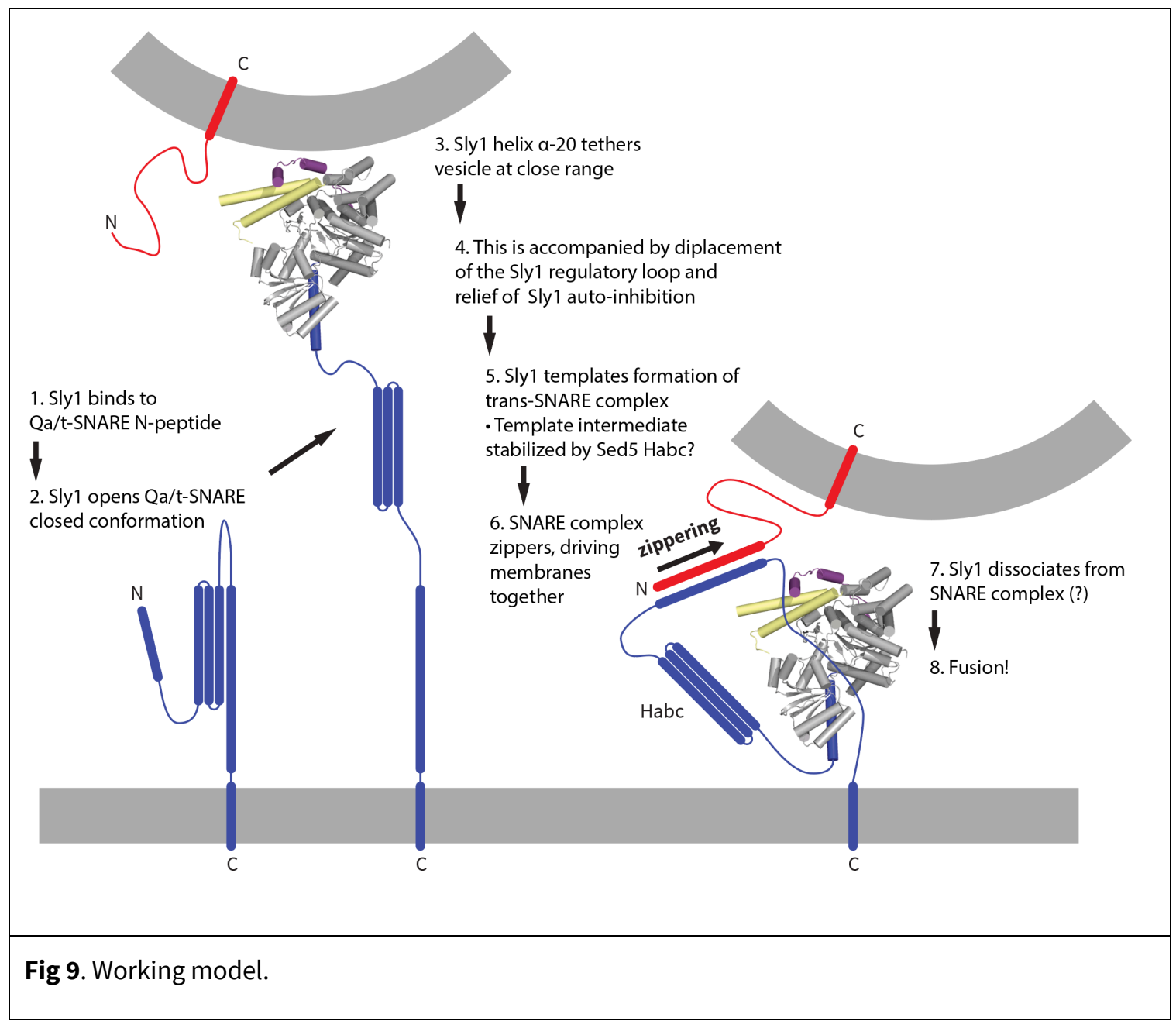


tethering function is closely coupled to selective catalysis of trans-SNARE complex assembly. Although the precise mechanism through which this occurs is not yet clear, structural data are suggestive. If the R-SNARE Sec22 binds to Sly1 in a configuration similar to the binding of the R-SNARE Nyv1 to Vps33 (Baker et al., 2015), then the open Sly1 loop should tether the incoming vesicle in an orientation optimal for capture of the vesicular R-SNARE. We therefore speculate that the close-range tethering mechanism serves not only to inspect incoming vesicle membranes and to trigger Sly1 activation, but to steer Sly1 into a spatial orientation that maximizes the likelihood of productive R-SNARE binding to domain 3a. When cis-SNARE complexes can form in competition with trans-complexes, tethering-defective Sly1 mutants exhibit profound fusion defects, even when tethering is stimulated by 4\% PEG (Figs. 6 and 7).

At the presynaptic nerve terminal the SM protein UNC-18/Munc18-1 seems to lock its Qa-SNARE, Syntaxin-1A, into a closed conformation. Another protein, UNC-13/Munc13-1 appears to be primarily responsible for opening syntaxin-1A (Richmond et al., 2001; Wang et al., 2017; Yang et al., 2015). However, it is unclear whether proteins homologous or analogous to UNC-13 are required for Qa-SNARE opening during other SNARE-mediated fusion events (Pei et al., 2009). Like Syntaxin-1A, the ER-Golgi Qa-SNARE Sed5 is normally autoinhibited. In contrast to Munc-18, Sly1 opens the closed Qa-SNARE (Demircioglu et al., 2014). However, Sly1 still stimulates function of Sed5 $\Delta \mathrm{Habc}$ mutants that cannot close. This was, perhaps, expected. Although all Qa (syntaxin-family) SNARE proteins have trihelical Habc domains, Habc domains are not always auto-inhibitory - yet SM proteins are still needed. For example Vam3, the Qa-SNARE of the yeast lysosomal vacuole, is constitutively open and its Habc domain lacks a groove that might bind to its SNARE domain, yet the SM protein Vps33 is still essential for fusion (Baker et al., 2015; Dulubova et al., 2001; Lobingier et al., 2014; Rieder and Emr, 1997; Seals et al., 2000). Thus, clamping the Qa-SNARE in a closed conformation, and opening the closed conformation, are pathway-specific elaborations rather than activities common to all SMs.

In addition to the above functions Sly1 has additional activities. Sly1 reduces the rate of SNARE complex disassembly by Sec17 and Sec18 (Lobingier et al., 2014). This is consistent with studies of Vps33 and Munc18-1, showing that these SMs protect assembled trans-SNARE complexes from premature disassembly by Sec17 and Sec18 (Lobingier et al., 2014; Prinslow 
et al., 2019; Schwartz et al., 2017; Song et al., 2017; Stepien et al., 2019; Xu et al., 2010; Duan et al., submitted). Thus we reiterate our previous suggestions (Lobingier et al., 2014; Schwartz et al., 2017) that SM proteins are, sensu stricto, enzymes.

Like all enzymes, SMs bind substrates (vesicular and target SNARE domains), placing them in a stereoselective orientation that reduces the kinetic barrier to formation of product (the trans-SNARE complex); the SMs then dissociate from the product to engage in additional cycles of catalysis (Jiao et al., 2018; Schwartz et al., 2017). Also as expected for true enzymes, SMs prevent off-pathway reactions (e.g., assembly of non-cognate SNARE complexes, or of cisrather than trans-complexes). They achieve this increase in specificity through kinetic partitioning within the forward assembly pathway (Hardy and Randall, 1991; Lai et al., 2017; Lambright et al., 1994; Peng and Gallwitz, 2002), and through kinetic proofreading of incorrect SNARE assemblies, since SMs selectively protect cognate SNARE complexes from premature disassembly by proofreading enzymes, while non-cognate complexes are efficiently disassembled (Choi et al., 2018; Lobingier et al., 2014; Prinslow et al., 2019; Schwartz et al., 2017; Song et al., 2017; Xu et al., 2010).

We were somewhat surprised to find that Sly1-dependent fusion driven by Sed5 $\Delta \mathrm{Habc}$ is slower than fusion driven by wild-type Sed5. Similarly, deletion of the Vam3 Habc domain causes a kinetic defect in homotypic vacuole fusion (Laage and Ungermann, 2001; Lürick et al., 2015; Pieren et al., 2010); but see (Wang et al., 2001). When we added soluble Habc domain to reactions containing Sed5 $\triangle \mathrm{Habc}$ RPLs, fusion activity was restored to wild-type or nearly wild-type levels (Fig. 8). The Habc domain of Sed5 therefore must have a positive function. What could this function be? Pieren et al. (2010) suggested that the Vam3 Habc domain might, through an interaction with Vps33, facilitate a transition from lipid mixing to content mixing. However, we have detected no signals consistent with the hypothesis that the Sed5 Habc domain influences the transition from lipid to content mixing. Experiments from the Zhang laboratory are more suggestive of an underlying mechanism. Using single-molecule force spectroscopy, they probed the formation and stability of template complexes consisting of neuronal SNAREs and the cognate SM Munc18-1. Formation of the SNARE-Munc18-1 template complex was almost an order of magnitude less efficient when Syntaxin lacked its N-terminal regulatory domain (the $\mathrm{N}$-peptide and Habc domain). In a striking parallel to our fusion 
bioRxiv preprint doi: https://doi.org/10.1101/2020.01.16.909630; this version posted January 16, 2020. The copyright holder for this preprint

(which was not certified by peer review) is the author/funder, who has granted bioRxiv a license to display the preprint in perpetuity. It is made available under aCC-BY-NC-ND 4.0 International license.

405 experiments (Figs. 8 and 9), addition of the soluble N-Habc domain rescued template complex formation and increased stability of the template complex by almost an order of magnitude (Jiao et al., 2018). The underlying structural basis for this stabilization is not yet understood. 


\section{MATERIALS \& METHODS}

Yeast strains, genetic tests, and microscopy. Yeast and E. coli strains are listed in Supplementary Table I. Viability assays were performed as described (Gao and Banfield, 2019).

Proteins. Full length SNAREs were expressed and purified as described in the companion manuscript. Constructs used to express mutant forms of Sed5 are listed in Supplementary Table 1. Sed5 mutants bearing transmembrane domains were expressed and purified as for full-length wild type Sed5. Soluble domains of Sed5 were expressed and purified as in the companion manuscript. Sly1 and its mutants were expressed and purified as described (Duan et al., submitted).

RPLs and Fusion assays. RPL lipid compositions, detailed methods for RPL preparation, and the in vitro fusion assay are described in the companion study (Duan et al., submitted). In this study additional SNARE topologies were tested, as described in the Results. The molar protein:phospholipid ratio for 4-SNARE liposomes was 1:1200, and 1:600 for Qabc and R SNARE RPLs. For certain experiments, as specified, reactions were set up with a non-standard order of reagent addition, and/or fusion was initiated by adding PEG, rather than by adding 425 Sly1 or its mutants.

\section{ACKNOWLEDGEMENTS}

We are grateful to Drs. R. Baker, I. Topalidou, and M. Zick for helpful advice and critical comments on the manuscript; and D. Beacham (Molecular Probes/Thermo Fisher) for gifts of fluorescent reagents. This work was funded by NIH/NIGMS R01 GM077349 and by University of Washington (AM), and the Hong Kong Research Council GRF 16101718 and AoE/M-05/12-2 (DB) 


\section{REFERENCES}

Angers, C. G., and A. J. Merz. 2011. New links between vesicle coats and Rab-mediated vesicle targeting. Semin Cell Dev Biol. 22:18-26.

Baker, R. W., P. D. Jeffrey, M. Zick, B. P. Phillips, W. T. Wickner, and F. M. Hughson. 2015. A direct role for the Sec1/Munc18-family protein Vps33 as a template for SNARE assembly. Science. 349:11111114.

Bombardier, J. P., and M. Munson. 2015. Three steps forward, two steps back: mechanistic insights into the assembly and disassembly of the SNARE complex. Curr Opin Chem Biol. 29:66-71.

Bracher, A., and W. Weissenhorn. 2002. Structural basis for the Golgi membrane recruitment of Sly1p by Sed5p. EMBO J. 21:6114-6124.

Carpp, L. N., L. F. Ciufo, S. G. Shanks, A. Boyd, and N. J. Bryant. 2006. The Sec1p/Munc18 protein Vps45p binds its cognate SNARE proteins via two distinct modes. J Cell Biol. 173:927-936.

Carr, C. M., and J. Rizo. 2010. At the junction of SNARE and SM protein function. Curr Opin Cell Biol. 22:488-495.

Choi, U. B., M. Zhao, K. I. White, R. A. Pfuetzner, L. Esquivies, Q. Zhou, and A. T. Brunger. 2018. NSFmediated disassembly of on- and off-pathway SNARE complexes and inhibition by complexin. Elife. 7

Dascher, C., R. Ossig, D. Gallwitz, and H. D. Schmitt. 1991. Identification and structure of four yeast genes (SLY) that are able to suppress the functional loss of YPT1, a member of the RAS superfamily. Mol Cell Biol. 11:872-885.

Demircioglu, F. E., P. Burkhardt, and D. Fasshauer. 2014. The SM protein Sly1 accelerates assembly of the ER-Golgi SNARE complex. Proc Natl Acad Sci U S A. 111:13828-13833.

Duan, M., R. L. Plemel, T. Takenaka, A. Lin, U. Natterman, J. Mima, E. A. Miller, and A. J. Merz. submitted. A gatekeeper helix activates the ER-Golgi SM protein Sly1 and directly mediates close-range vesicle tethering.

Dulubova, I., S. Sugita, S. Hill, M. Hosaka, I. Fernandez, T. C. Südhof, and J. Rizo. 1999. A conformational switch in syntaxin during exocytosis: role of munc18. EMBO J. 18:4372-4382.

Dulubova, I., T. Yamaguchi, D. Arac, H. Li, I. Huryeva, S. W. Min, J. Rizo, and T. C. Sudhof. 2003. Convergence and divergence in the mechanism of SNARE binding by Sec1/Munc18-like proteins. Proc Natl Acad Sci U S A. 100:32-37.

Dulubova, I., T. Yamaguchi, Y. Gao, S. W. Min, I. Huryeva, T. C. Sudhof, and J. Rizo. 2002. How Tlg2p/syntaxin 16 'snares' Vps45. EMBO J. 21:3620-3631.

Dulubova, I., T. Yamaguchi, Y. Wang, T. C. Sudhof, and J. Rizo. 2001. Vam3p structure reveals conserved and divergent properties of syntaxins. Nat Struct Biol. 8:258-264.

Fasshauer, D., R. B. Sutton, A. T. Brunger, and R. Jahn. 1998. Conserved structural features of the synaptic fusion complex: SNARE proteins reclassified as Q- and R-SNAREs. Proc Natl Acad Sci U S A. 95:15781-15786.

Fernandez, I., J. Ubach, I. Dulubova, X. Zhang, T. C. Sudhof, and J. Rizo. 1998. Three-dimensional structure of an evolutionarily conserved N-terminal domain of syntaxin 1A. Cell. 94:841-849.

Furgason, M. L., C. MacDonald, S. G. Shanks, S. P. Ryder, N. J. Bryant, and M. Munson. 2009. The Nterminal peptide of the syntaxin Tlg2p modulates binding of its closed conformation to Vps45p. Proc Natl Acad Sci U S A. 106:14303-14308.

Furukawa, N., and J. Mima. 2014. Multiple and distinct strategies of yeast SNAREs to confer the specificity of membrane fusion. Sci Rep. 4:4277.

Gao, G., and D. K. Banfield. 2019. Multiple features within the syntaxin Sed5p mediate its Golgi localization. Traffic.

Grabowski, R., and D. Gallwitz. 1997. High-affinity binding of the yeast cis-Golgi t-SNARE, Sed5p, to wildtype and mutant Sly1p, a modulator of transport vesicle docking. FEBS Lett. 411:169-172. 
Hanson, P. I., R. Roth, H. Morisaki, R. Jahn, and J. E. Heuser. 1997. Structure and conformational changes in NSF and its membrane receptor complexes visualized by quick-freeze/deep-etch electron microscopy. Cell. 90:523-535.

Hardy, S. J., and L. L. Randall. 1991. A kinetic partitioning model of selective binding of nonnative proteins by the bacterial chaperone SecB. Science. 251:439-443.

Jahn, R., and D. Fasshauer. 2012. Molecular machines governing exocytosis of synaptic vesicles. Nature. 490:201-207.

Jiao, J., M. He, S. A. Port, R. W. Baker, Y. Xu, H. Qu, Y. Xiong, Y. Wang, H. Jin, T. J. Eisemann, F. M. Hughson, and Y. Zhang. 2018. Munc18-1 catalyzes neuronal SNARE assembly by templating SNARE association. Elife. 7

Kosodo, Y., Y. Noda, and K. Yoda. 1998. Protein-protein interactions of the yeast Golgi t-SNARE Sed5 protein distinct from its neural plasma membrane cognate syntaxin 1. Biochem Biophys Res Commun. 250:212-216.

Laage, R., and C. Ungermann. 2001. The N-terminal domain of the t-SNARE Vam3p coordinates priming and docking in yeast vacuole fusion. Mol Biol Cell. 12:3375-3385.

Lai, Y., U. B. Choi, J. Leitz, H. J. Rhee, C. Lee, B. Altas, M. Zhao, R. A. Pfuetzner, A. L. Wang, N. Brose, J. Rhee, and A. T. Brunger. 2017. Molecular Mechanisms of Synaptic Vesicle Priming by Munc13 and Munc18. Neuron. 95:591-607.e10.

Lambright, D. G., S. Balasubramanian, S. M. Decatur, and S. G. Boxer. 1994. Anatomy and dynamics of a ligand-binding pathway in myoglobin: the roles of residues $45,60,64$, and 68 . Biochemistry. 33:5518-5525.

Lentz, B. R. 2007. PEG as a tool to gain insight into membrane fusion. Eur Biophys J. 36:315-326.

Lobingier, B. T., and A. J. Merz. 2012. Sec1/Munc18 protein Vps33 binds to SNARE domains and the quaternary SNARE complex. Mol Biol Cell. 23:4611-4622.

Lobingier, B. T., D. P. Nickerson, S. Y. Lo, and A. J. Merz. 2014. SM proteins Sly1 and Vps33 co-assemble with Sec17 and SNARE complexes to oppose SNARE disassembly by Sec18. Elife. 3:e02272.

Lürick, A., A. Kuhlee, C. Bröcker, D. Kümmel, S. Raunser, and C. Ungermann. 2015. The Habc domain of the SNARE Vam3 interacts with the HOPS tethering complex to facilitate vacuole fusion. J Biol Chem. 290:5405-5413.

Ma, L., A. A. Rebane, G. Yang, Z. Xi, Y. Kang, Y. Gao, and Y. Zhang. 2015. Munc18-1-regulated stage-wise SNARE assembly underlying synaptic exocytosis. Elife. 4

Misura, K. M., R. H. Scheller, and W. I. Weis. 2000. Three-dimensional structure of the neuronal-Sec1syntaxin 1a complex. Nature. 404:355-362.

Mitchison, T. J. 2019. Colloid osmotic parameterization and measurement of subcellular crowding. Mol Biol Cell. 30:173-180.

Munson, M., and F. M. Hughson. 2002. Conformational regulation of SNARE assembly and disassembly in vivo. J Biol Chem. 277:9375-9381.

Nichols, B. J., C. Ungermann, H. R. Pelham, W. T. Wickner, and A. Haas. 1997. Homotypic vacuolar fusion mediated by $\mathrm{t}-$ and $\mathrm{v}$-SNAREs. Nature. 387:199-202.

Nicholson, K. L., M. Munson, R. B. Miller, T. J. Filip, R. Fairman, and F. M. Hughson. 1998. Regulation of SNARE complex assembly by an N-terminal domain of the t-SNARE Sso1p. Nat Struct Biol. 5:793802.

Novick, P., R. Schekman, P. Novick, C. Field, and R. Schekman. 1979. Secretion and cell-surface growth are blocked in a temperature-sensitive mutant of Saccharomyces cerevisiae.

Identification of 23 complementation groups required for post-translational events in the yeast secretory pathway. Proc Natl Acad Sci U S A Cell. 76:1858-1862.

Ossig, R., C. Dascher, H. H. Trepte, H. D. Schmitt, and D. Gallwitz. 1991. The yeast SLY gene products, suppressors of defects in the essential GTP-binding Ypt1 protein, may act in endoplasmic reticulum-to-Golgi transport. Mol Cell Biol. 11:2980-2993.

Patterson, J. T. 1932. A New Type of Mottled-Eyed Drosophila Due to an Unstable Translocation. Genetics. 17:38-59. 
Pei, J., C. Ma, J. Rizo, and N. V. Grishin. 2009. Remote homology between Munc13 MUN domain and vesicle tethering complexes. J Mol Biol. 391:509-517.

Peng, R., and D. Gallwitz. 2002. Sly1 protein bound to Golgi syntaxin Sed5p allows assembly and contributes to specificity of SNARE fusion complexes. J Cell Biol. 157:645-655.

Peng, R., and D. Gallwitz. 2004. Multiple SNARE interactions of an SM protein: Sed5p/Sly1p binding is dispensable for transport. EMBO J. 23:3939-3949.

Pfeffer, S. R. 2017. Rab GTPases: master regulators that establish the secretory and endocytic pathways. Mol Biol Cell. 28:712-715.

Pieren, M., A. Schmidt, and A. Mayer. 2010. The SM protein Vps33 and the t-SNARE H(abc) domain promote fusion pore opening. Nat Struct Mol Biol. 17:710-717.

Prinslow, E. A., K. P. Stepien, Y. Z. Pan, J. Xu, and J. Rizo. 2019. Multiple factors maintain assembled trans-SNARE complexes in the presence of NSF and $\alpha$ SNAP. Elife. 8

Richmond, J. E., R. M. Weimer, and E. M. Jorgensen. 2001. An open form of syntaxin bypasses the requirement for UNC-13 in vesicle priming. Nature. 412:338-341.

Rieder, S. E., and S. D. Emr. 1997. A novel RING finger protein complex essential for a late step in protein transport to the yeast vacuole. Mol Biol Cell. 8:2307-2327.

Rizo, J., and T. C. Sudhof. 2012. The membrane fusion enigma: SNAREs, Sec1/Munc18 proteins, and their accomplices--guilty as charged? Annu Rev Cell Dev Biol. 28:279-308.

Schwartz, M. L., D. P. Nickerson, B. T. Lobingier, R. L. Plemel, M. Duan, C. G. Angers, M. Zick, and A. J. Merz. 2017. Sec17 ( $\alpha$-SNAP) and an SM-tethering complex regulate the outcome of SNARE zippering in vitro and in vivo. Elife. 6

Seals, D. F., G. Eitzen, N. Margolis, W. T. Wickner, and A. Price. 2000. A Ypt/Rab effector complex containing the Sec1 homolog Vps33p is required for homotypic vacuole fusion. Proc Natl Acad Sci U S A. 97:9402-9407.

Song, H., A. Orr, M. Duan, A. J. Merz, and W. Wickner. 2017. Sec17/Sec18 act twice, enhancing membrane fusion and then disassembling cis-SNARE complexes. Elife. 6

Stenmark, H. 2012. The Rabs: a family at the root of metazoan evolution. BMC Biol. 10:68.

Stepien, K. P., E. A. Prinslow, and J. Rizo. 2019. Munc18-1 is crucial to overcome the inhibition of synaptic vesicle fusion by $\alpha$ SNAP. Nat Commun. 10:4326.

Struthers, M. S., S. G. Shanks, C. MacDonald, L. N. Carpp, A. M. Drozdowska, D. Kioumourtzoglou, M. L. Furgason, M. Munson, and N. J. Bryant. 2009. Functional homology of mammalian syntaxin 16 and yeast Tlg2p reveals a conserved regulatory mechanism. J Cell Sci. 122:2292-2299.

Sudhof, T. C., and J. E. Rothman. 2009. Membrane fusion: grappling with SNARE and SM proteins. Science. 323:474-477.

Sutton, R. B., D. Fasshauer, R. Jahn, and A. T. Brunger. 1998. Crystal structure of a SNARE complex involved in synaptic exocytosis at 2.4 Å resolution. Nature. 395:347-353.

Togneri, J., Y. S. Cheng, M. Munson, F. M. Hughson, and C. M. Carr. 2006. Specific SNARE complex binding mode of the Sec1/Munc-18 protein, Sec1p. Proc Natl Acad Sci U S A. 103:17730-17735.

Toonen, R. F., and M. Verhage. 2003. Vesicle trafficking: pleasure and pain from SM genes. Trends Cell Biol. 13:177-186.

Ungar, D., and F. M. Hughson. 2003. SNARE protein structure and function. Annu Rev Cell Dev Biol. 19:493-517.

Wang, S., U. B. Choi, J. Gong, X. Yang, Y. Li, A. L. Wang, X. Yang, A. T. Brunger, and C. Ma. 2017. Conformational change of syntaxin linker region induced by Munc13s initiates SNARE complex formation in synaptic exocytosis. EMBO J. 36:816-829.

Wang, Y., I. Dulubova, J. Rizo, and T. C. Sudhof. 2001. Functional analysis of conserved structural elements in yeast syntaxin Vam3p. J Biol Chem. 276:28598-28605.

Weber, T., B. V. Zemelman, J. A. McNew, B. Westermann, M. Gmachl, F. Parlati, T. H. Sollner, and J. E. Rothman. 1998. SNAREpins: minimal machinery for membrane fusion. Cell. 92:759-772.

Xu, H., Y. Jun, J. Thompson, J. Yates, and W. Wickner. 2010. HOPS prevents the disassembly of transSNARE complexes by Sec17p/Sec18p during membrane fusion. EMBO J. 29:1948-1960. 
Yamaguchi, T., I. Dulubova, S. W. Min, X. Chen, J. Rizo, and T. C. Südhof. 2002. Sly1 binds to Golgi and ER syntaxins via a conserved N-terminal peptide motif. Dev Cell. 2:295-305.

Yang, B., M. Steegmaier, L. C. Gonzalez, and R. H. Scheller. 2000. nSec1 binds a closed conformation of syntaxin1A. J Cell Biol. 148:247-252.

Yang, X., S. Wang, Y. Sheng, M. Zhang, W. Zou, L. Wu, L. Kang, J. Rizo, R. Zhang, T. Xu, and C. Ma. 2015. Syntaxin opening by the MUN domain underlies the function of Munc13 in synaptic-vesicle priming. Nat Struct Mol Biol. 22:547-554.

Yoon, T. Y., and M. Munson. 2018. SNARE complex assembly and disassembly. Curr Biol. 28:R397-R401.

Yu, H., S. S. Rathore, C. Shen, Y. Liu, Y. Ouyang, M. H. Stowell, and J. Shen. 2015. Reconstituting Intracellular Vesicle Fusion Reactions: The Essential Role of Macromolecular Crowding. J Am Chem Soc. 137:12873-12883.

Zorman, S., A. A. Rebane, L. Ma, G. Yang, M. A. Molski, J. Coleman, F. Pincet, J. E. Rothman, and Y. Zhang. 2014. Common intermediates and kinetics, but different energetics, in the assembly of SNARE proteins. Elife. 3:e03348.

Zucchi, P. C., and M. Zick. 2011. Membrane fusion catalyzed by a Rab, SNAREs, and SNARE chaperones is accompanied by enhanced permeability to small molecules and by lysis. Mol Biol Cell. 22:46354646. 


\section{Supplementary Table S1. Yeast strains and plasmids used in this study}

\begin{tabular}{|c|c|c|}
\hline Strain/Plasmid & Genotype/description & Reference/source \\
\hline \multicolumn{3}{|c|}{ Yeast Strains } \\
\hline SARY4487 & sly1::NAT sed5::KanMX4 pRS416::SLY1 SED5 (URA3) & Banfield collection \\
\hline BY4741 & MATa his3 $\Delta 1$ leu2 $\Delta 0$ met $15 \Delta 0$ ura $3 \Delta 0$ & Banfield collection \\
\hline \multicolumn{3}{|c|}{\begin{tabular}{|l} 
Yeast expression plasmids \\
\end{tabular}} \\
\hline GBp1B9 & pRS416::SLY1 SED5 (URA3) & Banfield collection \\
\hline GBp12A4 & pRS415::SLY1 & Banfield collection \\
\hline GBp12B5 & pRS425::SLY1 & Banfield collection \\
\hline GBp12B2 & pRS415::sly1-20 & Banfield collection \\
\hline GBp12B7 & pRS425::sly1-20 & Banfield collection \\
\hline GBp12A6 & pRS413::SED5 & Banfield collection \\
\hline GBp12A8 & pRS413::sed5 $\Delta N(\Delta 1-21)$ & Banfield collection \\
\hline GBp12D5 & pRS413::sed5 $\Delta N-H a b c(\Delta 1-180)$ & Banfield collection \\
\hline GBp12D7 & pRS413::sed5 $\Delta H a b c(\Delta 51-180)$ & Banfield collection \\
\hline GBp13G2 & pRS413::sed5 $\Delta N-H a b c(\Delta 1-180)+T P I 1 p-s e d 5(1-180)-C Y C 1 t$ & Banfield collection \\
\hline GBp13G4 & pRS413::sed5 $\Delta H a b c(\Delta 51-180)+$ TPI1p-sed5(1-180)-CYC1t & Banfield collection \\
\hline GBp13G8 & pRS413::sed5 $\Delta N-H a b c(\Delta 1-180)+$ TPI1p-sed5(51-180)-CYC1t & Banfield collection \\
\hline GBp13H2 & pRS413::sed5 $\Delta H a b c(\Delta 51-180)+$ TPI1p-sed5(51-180)-CYC1t & Banfield collection \\
\hline GBp5D1 & pRS416::mNeon-SED5 & Banfield collection \\
\hline GBp13H9 & pRS416::EmCritine-sed5 $\Delta 21$ & Banfield collection \\
\hline GBp12E6 & pRS413:: mNeon-sed5 $\Delta N-H a b c(\Delta 1-180)$ & Banfield collection \\
\hline GBp12E4 & pRS413:: mNeon-sed5 $\Delta H a b c(\Delta 51-180)$ & Banfield collection \\
\hline \multicolumn{3}{|c|}{ E. coli SNARE expression plasmids } \\
\hline AMP1792 & pET-30::His $6^{-}(3 C)-S E D 5$ & (Furukawa and Mima, 2014) \\
\hline AMP1875 & pET-30::His6-(3C)-SED5 $N N(\Delta 1-21)$ & This study \\
\hline AMP1962 & pET-30::His6-(3C)-SED5 $\Delta H a b c(\Delta 51-180)$ & This study \\
\hline AMP1963 & pET-30::His ${ }_{6}-(3 C)-S E D 5 \Delta N-H a b c(\Delta 1-21, \Delta 51-180)$ & This study \\
\hline AMP1961 & pET-30::His $6_{-}(3 C)-S E D 5 H a b c(22-210)$ & Duan et al. (submitted) \\
\hline AMP1960 & pET-30::His $6-(3 C)-S E D 5 N-H a b c(1-210)$ & Duan et al. (submitted) \\
\hline AMP2020??? & pET-30::His6-(3C)-Bos1 (Q153D) & (Furukawa and Mima, 2014; modified) \\
\hline AMP1794??? & pET-30::His6-(3C)-Bet1 (with corrected missense mutation) & (Furukawa and Mima, 2014) \\
\hline AMP1795 & pET-41::GST-His $6^{-}(3 C)-S e c 22$ & (Furukawa and Mima, 2014) \\
\hline AMP1687 & pST50Trc1::Sec22 $2_{S N}(120-188)-(3 C)-$ sfGFP-His $_{8}$ & This study \\
\hline \multicolumn{3}{|c|}{ E. coli SNARE chaperone expression plasmids } \\
\hline AMP1547 & pTYB12::intein-CBD-SEC17 & (Schwartz and Merz, 2009) \\
\hline AMP77??? & pQE9::His6-SEC18 & (Haas and Wickner 1996)/J. Rothman \\
\hline \multicolumn{3}{|c|}{ E. coli SLY1 expression plasmids } \\
\hline AMP1649(pBL51) & pHIS-Parallel1::His-(TEV)-Sly1 & (Lobingier and Merz, 2014) \\
\hline AMP1651 & pHIS-Parallel1::His ${ }_{6}-(T E V)-S l y 1-20$ & Duan et al. (submitted) \\
\hline AMP1652 & pHIS-Parallel1::His6-(TEV)-Sly1(T559I) & Duan et al. (submitted) \\
\hline AMP1654 & pHIS-Parallel1::His6-(TEV)-Sly1 1 loop & Duan et al. (submitted) \\
\hline AMP1932 & pHIS-Parallel1::His ${ }_{6}-(T E V)-S l y 1-p a 21$ & Duan et al. (submitted) \\
\hline AMP1933 & pHIS-Parallel1::His ${ }_{6}-(T E V)-S l y 1-20-p a 21$ & Duan et al. (submitted) \\
\hline \multicolumn{3}{|c|}{ E. coli miscellaneous expression plasmids } \\
\hline AMP1881 & pET-49::GST-His6-(3C) & Duan et al. (submitted) \\
\hline AMP2019 & pET-30::His8-HRV3C (protease) & Duan et al. (submitted) \\
\hline AMP2016 & pET-49::GST-His $6_{6}$ (thrombin)-HRV3C (pProtease) & Duan et al. (submitted) \\
\hline$? ? ?$ & Our homemade TEV expression plasmid & Duan et al. (submitted) \\
\hline
\end{tabular}


bioRxiv preprint doi: https://doi.org/10.1101/2020.01.16.909630; this version posted January 16, 2020. The copyright holder for this preprint (which was not certified by peer review) is the author/funder, who has granted bioRxiv a license to display the preprint in perpetuity. It is made available under aCC-BY-NC-ND 4.0 International license.

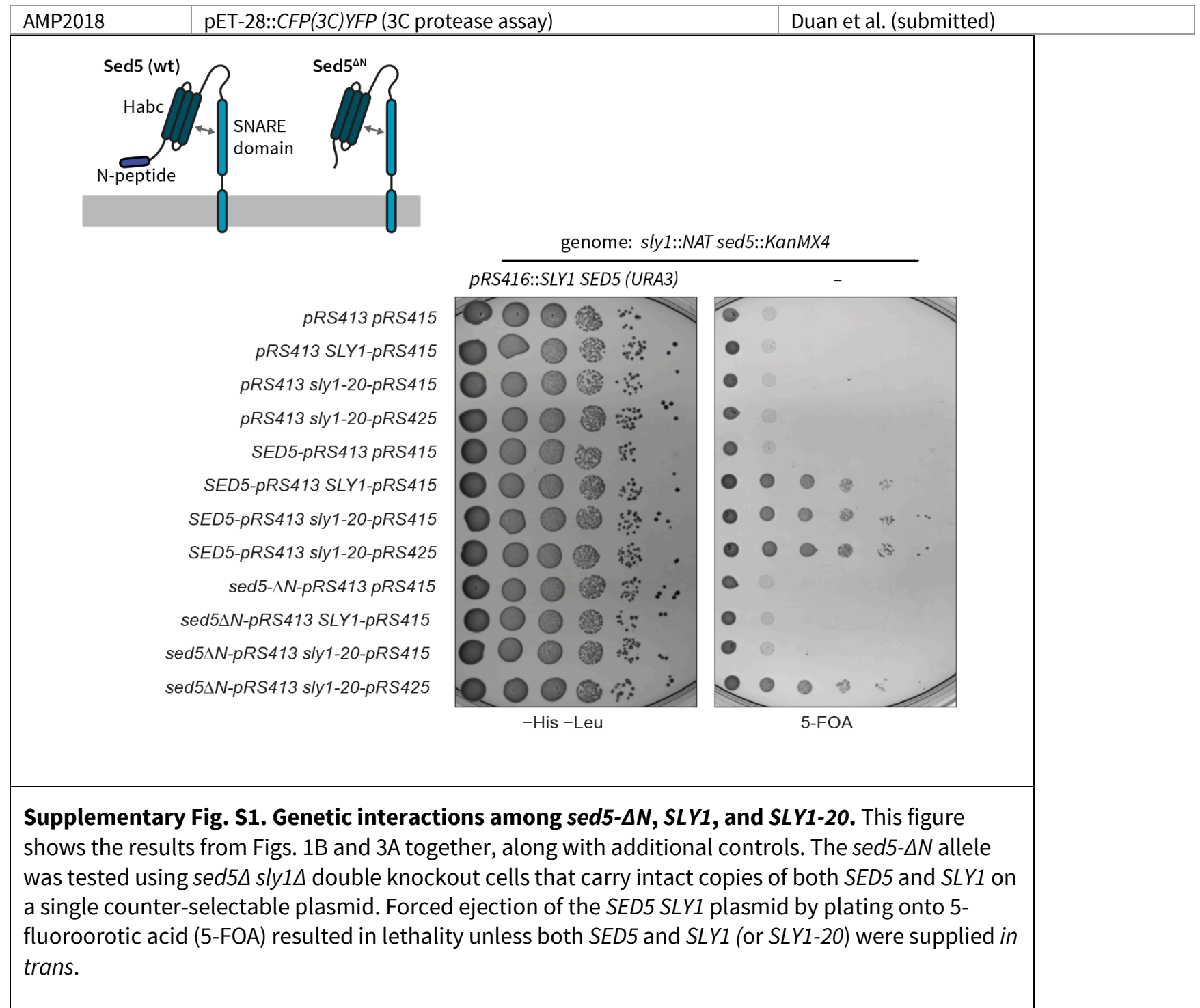




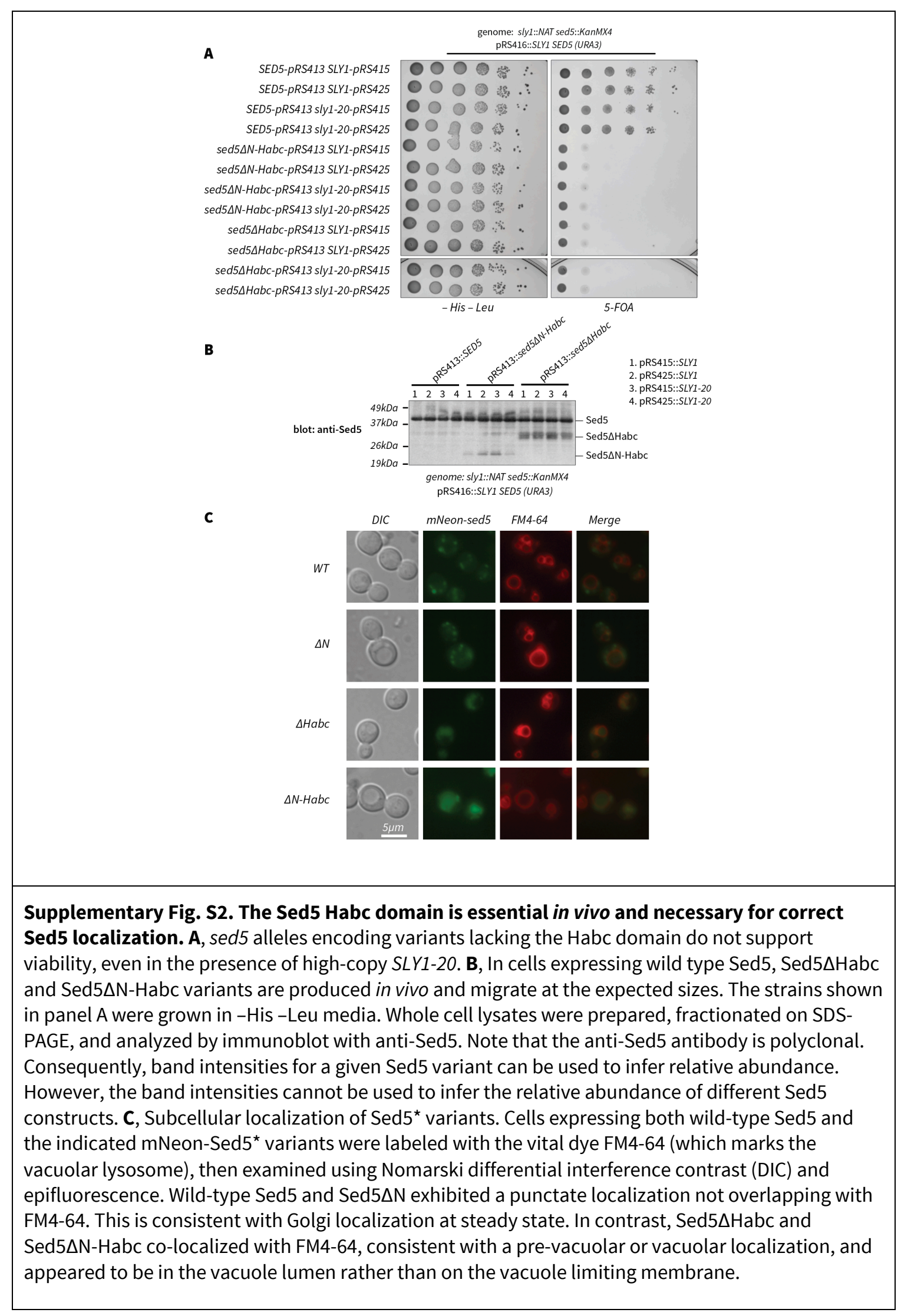




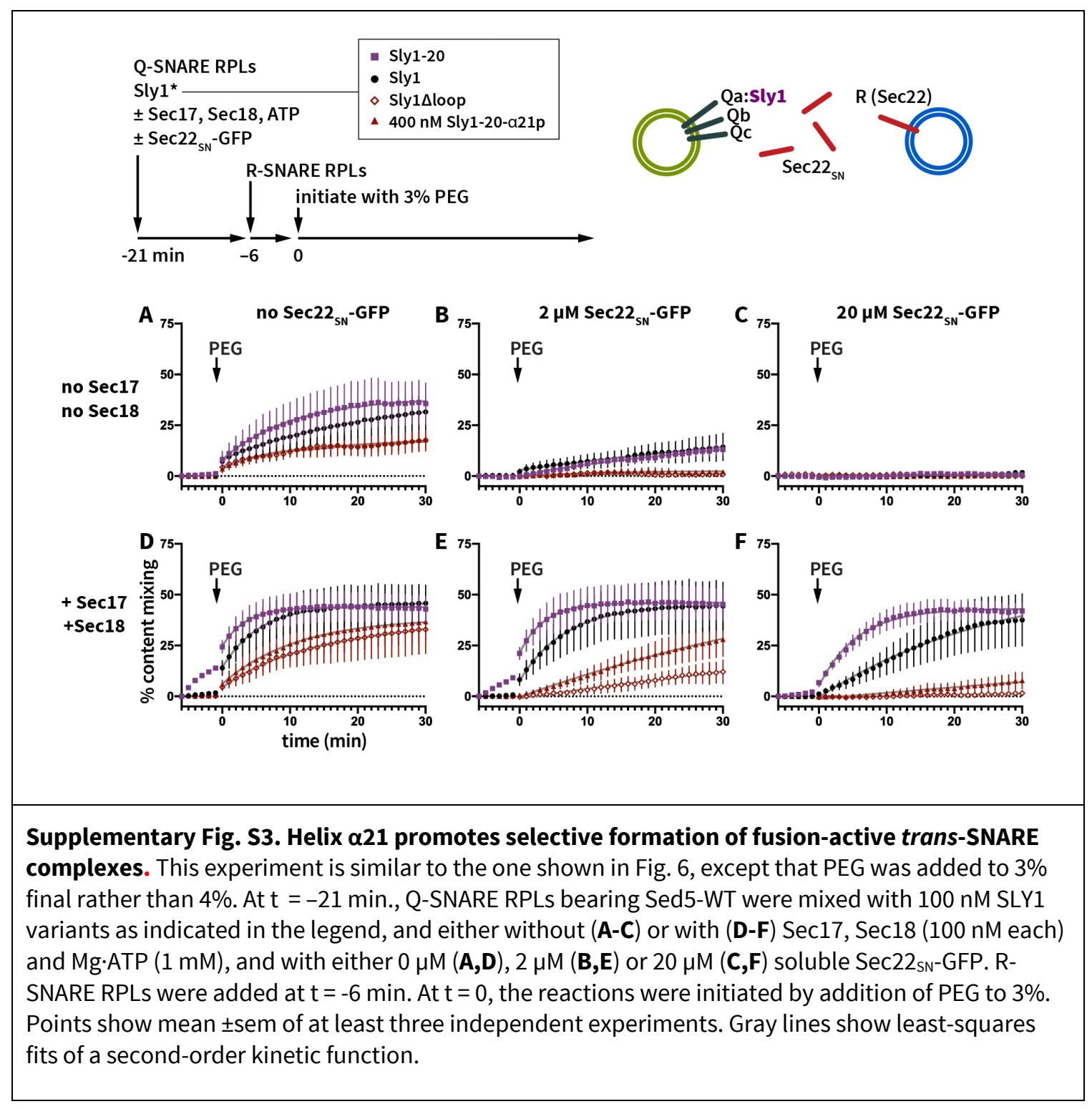


bioRxiv preprint doi: https://doi.org/10.1101/2020.01.16.909630; this version posted January 16,2020 . The copyright holder for this preprint (which was not certified by peer review) is the author/funder, who has granted bioRxiv a license to display the preprint in perpetuity. It is made available under aCC-BY-NC-ND 4.0 International license.

A

\begin{tabular}{|ll|}
\hline genome: sly1::NAT sed5::KanMX4 & 1. pRS415::SLY1 \\
pRS416::SLY1 SED5 (URA3) & 2. PRS425::SLY1 \\
& 3. pRS415::SLY1-20 \\
blot: anti-Sed5 & 4. pRS425::SLY1-20 \\
\hline
\end{tabular}
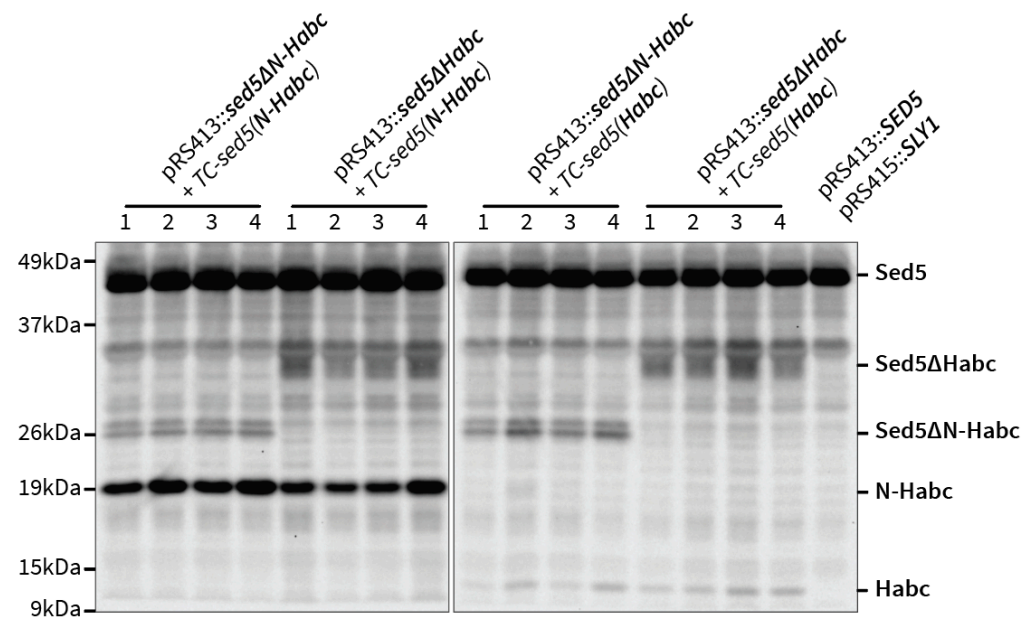

B

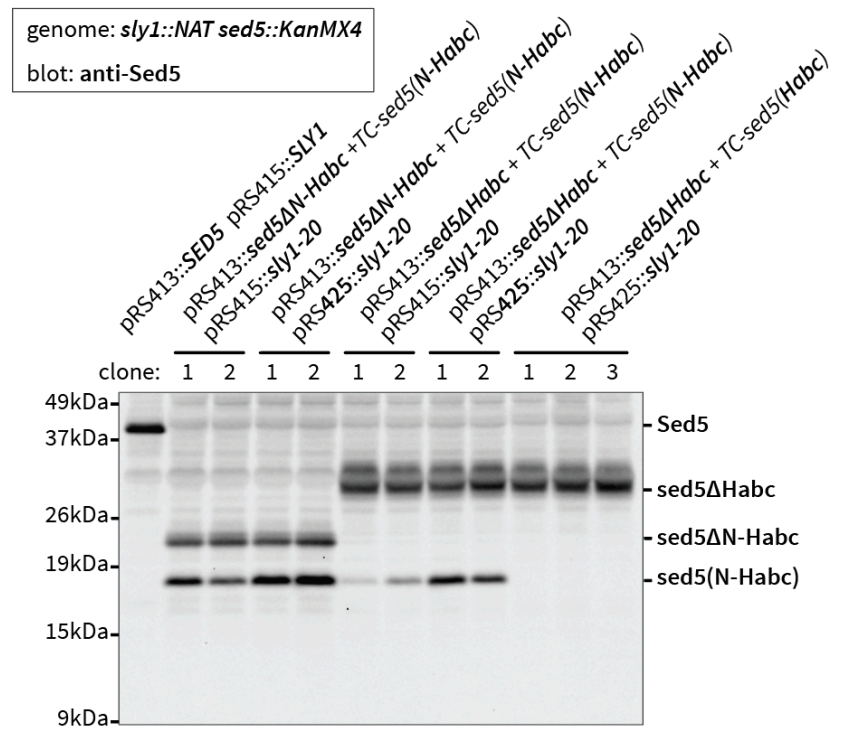

Supplementary Fig. S4. Co-expression of mutant Sed5 proteins and N-terminal Sed5 fragments. Wholecell lysates from the indicated strains were prepared, separated by SDS-PAGE, and immunoblotted with antiSed5. Panel A shows expression in strains containing a counterselectable SLY1 SED5 balancer plasmid. Panel B shows expression in strains harboring SLY1-20 on single-copy (pRS415) or multicopy (pRS425) plasmids following ejection of the SLY1 SED5 balancer plasmid. 